\title{
Spectral shift functions and Dirichlet-to-Neumann maps
}

\author{
Jussi Behrndt ${ }^{1}$ (D) Fritz Gesztesy ${ }^{2}$. \\ Shu Nakamura ${ }^{3}$
}

Received: 3 February 2017 / Revised: 2 August 2017 / Published online: 23 September 2017

(C) The Author(s) 2017. This article is an open access publication

\begin{abstract}
The spectral shift function of a pair of self-adjoint operators is expressed via an abstract operator-valued Titchmarsh-Weyl $m$-function. This general result is applied to different self-adjoint realizations of second-order elliptic partial differential operators on smooth domains with compact boundaries and Schrödinger operators with compactly supported potentials. In these applications the spectral shift function is determined in an explicit form with the help of (energy parameter dependent) Dirichletto-Neumann maps.
\end{abstract}

Mathematics Subject Classification Primary 35J10 - 35J15 - 47A10 - 47A40 * 47B25; Secondary 35P20 $\cdot 35 \mathrm{P} 25 \cdot 47 \mathrm{~A} 55 \cdot 47 \mathrm{~B} 10 \cdot 47 \mathrm{~F} 05 \cdot 81 \mathrm{Q} 10$

Communicated by Y. Giga.

$凶$ Jussi Behrndt

behrndt@tugraz.at

http://www.math.tugraz.at/ behrndt/

Fritz Gesztesy

Fritz_Gesztesy@baylor.edu

http://www.baylor.edu/math/index.php?id=935340

Shu Nakamura

shu@ms.u-tokyo.ac.jp

http://www.ms.u-tokyo.ac.jp/ shu/

1 Institut für Numerische Mathematik, Technische Universität Graz, Steyrergasse 30, 8010 Graz, Austria

2 Department of Mathematics, Baylor University, One Bear Place \#97328, Waco, TX 76798-7328, USA

3 Graduate School of Mathematical Sciences, University of Tokyo, 3-8-1, Komaba, Meguro-ku, Tokyo 153-8914, Japan 


\section{Introduction}

Let $A$ and $B$ be self-adjoint operators in a separable Hilbert space $\mathfrak{H}$ and assume that the $m$-th powers of their resolvents differ by a trace class operator,

$$
\left[\left(B-z I_{\mathfrak{H}}\right)^{-m}-\left(A-z I_{\mathfrak{H}}\right)^{-m}\right] \in \mathfrak{S}_{1}(\mathfrak{H}), \quad z \in \rho(A) \cap \rho(B),
$$

for some odd integer $m \in \mathbb{N}$. It is known that in this case there exists a real-valued function $\xi \in L_{\text {loc }}^{1}(\mathbb{R})$ such that $\int_{\mathbb{R}}|\xi(\lambda)|(1+|\lambda|)^{-(m+1)} d \lambda<\infty$ and the trace formula

$$
\operatorname{tr}_{\mathfrak{H}}(\varphi(B)-\varphi(A))=\int_{\mathbb{R}} \varphi^{\prime}(\lambda) \xi(\lambda) d \lambda
$$

holds for all suitable smooth functions $\varphi: \mathbb{R} \rightarrow \mathbb{C}$ such that $[\varphi(B)-\varphi(A)] \in \mathfrak{S}_{1}(\mathfrak{H})$. The function $\xi$ in (1.2) is called a spectral shift function of the pair $\{A, B\}$. Note that for $\varphi(\lambda)=(\lambda-z)^{-m}$ one has $[\varphi(B)-\varphi(A)] \in \mathfrak{S}_{1}(\mathfrak{H})$ according to (1.1) and the trace formula (1.2) takes the special form

$$
\operatorname{tr}_{\mathfrak{H}}\left(\left(B-z I_{\mathfrak{H}}\right)^{-m}-\left(A-z I_{\mathfrak{H}}\right)^{-m}\right)=-m \int_{\mathbb{R}} \frac{\xi(\lambda) d \lambda}{(\lambda-z)^{m+1}}
$$

Historically the trace formula (1.2) was first proposed and verified on a formal level by Lifshitz for the case that $[B-A]$ is a finite-rank operator in [51] (see also [52]), and shortly afterwards in [44] Krein proved (1.2) rigorously in the more general case $[B-A] \in \mathfrak{S}_{1}(\mathfrak{H})$ for all $C^{1}$-functions $\varphi$ with derivatives in the Wiener class. Furthermore, in [44] it was shown how the spectral shift function $\xi$ can be computed with the help of the perturbation determinant corresponding to the pair $\{A, B\}$. For pairs of unitary operators and thus via Cayley transforms for the case $m=1$ in (1.1) the spectral shift function and the trace formula were obtained later by Krein in [45]. Afterwards in [43] the more general case $m>1$ in (1.1) for self-adjoint operators $A$ and $B$ with $\rho(A) \cap \rho(B) \cap \mathbb{R} \neq \varnothing$ was discussed by Koplienko, and for odd integers $m$ in (1.1) and arbitrary self-adjoint operators $A$ and $B$ see [74] by Yafaev or [73, Chapter 8, §11] and [76, Chapter 0, Theorem 9.4]. We also mention that the spectral shift function is closely connected with the scattering matrix via the famous Birman-Krein formula from [11,12]. For more details on the history, development and multifaceted applications of the spectral shift function in mathematical analysis we refer the reader to the survey papers $[13,16,17]$, the standard monographs $[73,76]$, and, for instance, to $[14,19,24,26,27,31,46,47,67,70]$ and the more recent contributions $[1,25,30,39,40,42,48,55,56,64-66,68,75]$.

The main objective of the present paper is to prove a representation formula for the spectral shift function in terms of an abstract Titchmarsh-Weyl $m$-function of two self-adjoint operators satisfying the condition (1.1), and to apply this result to different self-adjoint realizations of second-order elliptic PDEs and Schrödinger operators with compactly supported potentials. In these applications the abstract Titchmarsh-Weyl $m$-function will turn out to be the energy dependent Neumann-to-Dirichlet map or 
Dirichlet-to-Neumann map associated to the elliptic differential expression and the Schrödinger operators on an interior and exterior domain, respectively.

More precisely, assume that $A$ and $B$ are self-adjoint operators in a separable Hilbert space $\mathfrak{H}$ and consider the underlying closed symmetric operator

$$
S f:=A f=B f, \quad \operatorname{dom}(S):=\{f \in \operatorname{dom}(A) \cap \operatorname{dom}(B) \mid A f=B f\},
$$

which for convenience we assume is densely defined. We emphasize that neither $A$ nor $B$ needs to be semibounded in our approach. However, we first impose an implicit sign condition on the perturbation by assuming

$$
\left(A-\mu_{0} I_{\mathfrak{H}}\right)^{-1} \geq\left(B-\mu_{0} I_{\mathfrak{H}}\right)^{-1}
$$

for some $\mu_{0} \in \rho(A) \cap \rho(B) \cap \mathbb{R}$; in the semibounded case the condition (1.3) is equivalent to $A \leq B$ interpreted in the sense of the corresponding quadratic forms. We then make use of the concept of quasi boundary triples in extension theory of symmetric operators from [2,3] and construct an operator $T$ such that $\bar{T}=S^{*}$ and two boundary mappings $\Gamma_{0}, \Gamma_{1}: \operatorname{dom}(T) \rightarrow \mathcal{G}$, where $\mathcal{G}$ is an auxiliary Hilbert space, such that

$$
A=T \uparrow \operatorname{ker}\left(\Gamma_{0}\right) \quad \text { and } \quad B=T \uparrow \operatorname{ker}\left(\Gamma_{1}\right)
$$

see Proposition 2.4 and Sect. 2 for more details. To such a quasi boundary triple $\left\{\mathcal{G}, \Gamma_{0}, \Gamma_{1}\right\}$ one associates the $\gamma$-field and Weyl function (or abstract TitchmarshWeyl $m$-function) $M$ which are defined by

$$
\gamma(z) \Gamma_{0} f_{z}=f_{z} \quad \text { and } \quad M(z) \Gamma_{0} f_{z}=\Gamma_{1} f_{z}, \quad f_{z} \in \operatorname{ker}\left(T-z I_{\mathfrak{H}}\right), \quad z \in \rho(A),
$$

respectively. Very roughly speaking the values $M(z), z \in \rho(A)$, of the function $M$ map abstract Dirichlet boundary values to abstract Neumann boundary values, or vice versa, and hence the Weyl function $M$ associated to a quasi boundary triple can be viewed as an abstract analog of the (energy parameter dependent) Dirichlet-to-Neumann map. The resolvents of $A$ and $B$ are related with the $\gamma$-field and Weyl function via the useful Krein-type formula

$$
\left(B-z I_{\mathfrak{H}}\right)^{-1}-\left(A-z I_{\mathfrak{H}}\right)^{-1}=-\gamma(z) M(z)^{-1} \gamma(\bar{z})^{*}, \quad z \in \rho(A) \cap \rho(B) .
$$

In our main result, Theorem 4.1, in the abstract part of this paper we provide sufficient $\mathfrak{S}_{p}$-type conditions on the $\gamma$-field and Weyl function of the quasi boundary triple $\left\{\mathcal{G}, \Gamma_{0}, \Gamma_{1}\right\}$ such that (1.1) is satisfied with $m=2 k+1$ and conclude that for any orthonormal basis $\left(\varphi_{j}\right)_{j \in J}$ in $\mathcal{G}$ (with $J \subseteq \mathbb{N}$ an appropriate index set), the function

$$
\xi(\lambda)=\sum_{j \in J} \lim _{\varepsilon \downarrow 0} \pi^{-1}\left(\operatorname{Im}(\log (\overline{M(\lambda+i \varepsilon)})) \varphi_{j}, \varphi_{j}\right)_{\mathcal{G}} \quad \text { for a.e. } \lambda \in \mathbb{R}
$$


is a spectral shift function for the pair $\{A, B\}$ such that $\xi(\lambda)=0$ in an open neighborhood of $\mu_{0}$. In particular, the trace formula

$$
\operatorname{tr}_{\mathfrak{H}}\left(\left(B-z I_{\mathfrak{H}}\right)^{-(2 k+1)}-\left(A-z I_{\mathfrak{H}}\right)^{-(2 k+1)}\right)=-(2 k+1) \int_{\mathbb{R}} \frac{\xi(\lambda) d \lambda}{(\lambda-z)^{2 k+2}}
$$

is valid for all $z \in \rho(A) \cap \rho(B)$. Furthermore, if (1.1) is satisfied with $m=1$ then according to Corollary 4.2 the imaginary part of the logarithm of $z \mapsto \overline{M(z)}$ is a trace class valued Nevanlinna (or Riesz-Herglotz) function on the open upper half-plane $\mathbb{C}_{+}$(and hence admits nontangential limits for a.e. $\lambda \in \mathbb{R}$ from $\mathbb{C}_{+}$in the trace class topology), and the spectral shift function in (1.5) has the form

$$
\xi(\lambda)=\lim _{\varepsilon \downarrow 0} \pi^{-1} \operatorname{tr}_{\mathcal{G}}(\operatorname{Im}(\log (\overline{M(\lambda+i \varepsilon)}))) \quad \text { for a.e. } \lambda \in \mathbb{R} \text {. }
$$

Since $z \mapsto \log (\overline{M(z)})$ is a Nevanlinna function it follows that the values of the spectral shift function $\xi$ in (1.5) and (1.6) are nonnegative for a.e. $\lambda \in \mathbb{R}$; this is rooted in the sign condition (1.3). In a second step we weaken the sign condition (1.3) and extend our representation of the spectral shift function to more general perturbations in the end of Sect. 4. We point out that the key difficulty in the proof of (1.5) and (1.6) is to ensure the existence of the limits on the right hand side of (1.5) and the trace class property of the function $\operatorname{Im}(\log (\bar{M}))$ in the case $k=0$, respectively, which are indispensable for (1.5) and (1.6). These problems are investigated separately in Sect. 3 on the logarithm of operator-valued Nevanlinna functions, where special attention is paid to the analytic continuation by reflection with respect to open subsets of the real line. We also mention that for the special case where (1.1) is a rank one or finite-rank operator and $m=1$, our representation for the spectral shift function coincides with the one in $[7,49]$. Furthermore, for $m=1$ in (1.1) a formula for the spectral shift function via a perturbation determinant involving boundary parameters and the Weyl function in the context of ordinary boundary triples was shown recently in [56] (see also [55]). We remark that our abstract result can also be formulated and remains valid in the special situation that the quasi boundary triple $\left\{\mathcal{G}, \Gamma_{0}, \Gamma_{1}\right\}$ is a generalized or ordinary boundary triple in the sense of [18,21-23,32].

Our main reason to provide the general result in Sect. 4 for the spectral shift function in terms of the abstract notion of quasi boundary triples and their Weyl functions is its convenient applicability to various PDE situations, see also [2-6,8] for other related applications of quasi boundary triples in PDE problems. In Sect. 5 we consider a formally symmetric uniformly elliptic second-order partial differential expression $\mathcal{L}$ with smooth coefficients on a bounded or unbounded domain in $\mathbb{R}^{n}, n \geq 2$, with compact boundary, and two self-adjoint realizations $A_{\beta_{0}}$ and $A_{\beta_{1}}$ of $\mathcal{L}$ subject to Robin boundary conditions $\beta_{p} \gamma_{D} f=\gamma_{N} f$, where $\gamma_{D}$ and $\gamma_{N}$ denote the Dirichlet and Neumann trace operators, and $\beta_{p} \in C^{1}(\partial \Omega), p=0,1$, are real-valued functions. It then turns out that the Robin realizations $A_{\beta_{0}}$ and $A_{\beta_{1}}$ satisfy

$$
\left[\left(A_{\beta_{1}}-z I_{L^{2}(\Omega)}\right)^{-(2 k+1)}-\left(A_{\beta_{0}}-z I_{L^{2}(\Omega)}\right)^{-(2 k+1)}\right] \in \mathfrak{S}_{1}\left(L^{2}(\Omega)\right)
$$


for all $k \in \mathbb{N}_{0}, k \geq(n-3) / 4$, and $z \in \rho\left(A_{\beta_{0}}\right) \cap \rho\left(A_{\beta_{1}}\right)$, and for any orthonormal basis $\left(\varphi_{j}\right)_{j \in J}$ in $L^{\overline{2}}(\partial \Omega)$, the function

$$
\begin{aligned}
& \xi(\lambda)=\sum_{j \in J} \lim _{\varepsilon \downarrow 0} \pi^{-1}\left(\left(\operatorname{Im}\left(\log \left(\mathcal{M}_{1}(\lambda+i \varepsilon)\right)-\log \left(\mathcal{M}_{0}(\lambda+i \varepsilon)\right)\right)\right) \varphi_{j}, \varphi_{j}\right)_{L^{2}(\partial \Omega)} \\
& \quad \text { for a.e. } \lambda \in \mathbb{R},
\end{aligned}
$$

is a spectral shift function for the pair $\left\{A_{\beta_{0}}, A_{\beta_{1}}\right\}$, where

$$
\mathcal{M}_{p}(z)=\left(\beta-\beta_{p}\right)^{-1}\left(\beta_{p} \overline{\mathcal{N}(z)}-I_{L^{2}(\partial \Omega)}\right)\left(\beta \overline{\mathcal{N}(z)}-I_{L^{2}(\partial \Omega)}\right)^{-1}, \quad z \in \mathbb{C} \backslash \mathbb{R},
$$

$\beta \in \mathbb{R}$ is such that $\beta_{p}(x)<\beta$ for all $x \in \partial \Omega$, and $\mathcal{N}(z)$ denotes the (z-dependent) Neumann-to-Dirichlet map that assigns Neumann boundary values of solutions $f_{z} \in$ $H^{2}(\Omega)$ of $\mathcal{L} f_{z}=z f_{z}, z \in \mathbb{C} \backslash \mathbb{R}$, onto their Dirichlet boundary values. We note that the trace class property (1.7) was shown in [4,34] for the case $k=0$ and in [6] for $k \geq 1$. Moreover, in the case $k=0$, that is, $n=2$ or $n=3$, it follows from (1.6) that the spectral shift function in (1.8) has the form

$\xi(\lambda)=\lim _{\varepsilon \downarrow 0} \pi^{-1} \operatorname{tr}_{L^{2}(\partial \Omega)}\left(\operatorname{Im}\left(\log \left(\mathcal{M}_{1}(\lambda+i \varepsilon)\right)-\log \left(\mathcal{M}_{0}(\lambda+i \varepsilon)\right)\right)\right)$ for a.e. $\lambda \in \mathbb{R}$.

In our second example, presented in Sect. 6, we consider a Schrödinger operator $B=-\Delta+V$ with a compactly supported potential $V \in L^{\infty}\left(\mathbb{R}^{n}\right)$. Here we split the Euclidean space $\mathbb{R}^{n}$ and the Schrödinger operator via a multi-dimensional Glazman decomposition and consider the orthogonal sum $B_{D}=B_{+} \oplus C$ of the Dirichlet realizations of $-\Delta+V$ in $L^{2}\left(\mathcal{B}_{+}\right)$and $L^{2}\left(\mathcal{B}_{-}\right)$, where $\mathcal{B}_{+}$is a sufficiently large ball which contains $\operatorname{supp}(V)$ and $\mathcal{B}_{-}:=\mathbb{R}^{n} \backslash \overline{\mathcal{B}}_{+}$. Similarly, the unperturbed operator $A=-\Delta$ is decoupled and compared with the orthogonal sum $A_{D}=A_{+} \oplus C$ of the Dirichlet realizations of $-\Delta$ in $L^{2}\left(\mathcal{B}_{+}\right)$and $L^{2}\left(\mathcal{B}_{-}\right)$. Our abstract result applies to the pairs $\left\{B, B_{D}\right\}$ and $\left\{A, A_{D}\right\}$, whenever $k>(n-2) / 4, n \in \mathbb{N}, n \geq 2$, and yields an explicit formula for their spectral shift functions $\xi_{B}$ and $\xi_{A}$ in terms of the (z-dependent) Dirichlet-to-Neumann maps associated to $-\Delta$ and $-\Delta+V$ on $\mathcal{B}_{+}$and $\mathcal{B}_{-}$. Since the spectra of the Dirichlet realizations $A_{+}=-\Delta$ and $B_{+}=-\Delta+V$ on the bounded domain $\mathcal{B}_{+}$are both discrete and bounded from below, the difference of their eigenvalue counting functions is a spectral shift function $\xi_{+}$for the pair $\left\{A_{+}, B_{+}\right\}$, and hence also for the pair $\left\{A_{D}, B_{D}\right\}$. Then it follows that the function

$$
\xi(\lambda)=\xi_{A}(\lambda)-\xi_{B}(\lambda)+\xi_{+}(\lambda) \quad \text { for a.e. } \lambda \in \mathbb{R},
$$

is a spectral shift function for the original pair $\{A, B\}$ (cf. Theorem 6.1). We also mention that the trace class property of the resolvent differences of $A$ and $A_{D}$, and $B$ and $B_{D}$ goes back to Birman [9] and Grubb [33], and that similar decoupling methods are often used in scattering theory, see, for instance, [20] or [71] for a slighty more abstract and general framework.

The applications in Sects. 5 and 6 serve as typical examples for the abstract formalism and results in Sect. 4. In this context we mention that one may compare in a 
similar form as in Sect. 5 the Dirichlet realization with the Neumann, or other selfadjoint Robin realizations of an elliptic partial differential expression, and that in principle also higher-order differential expressions with smooth coefficients could be considered. We refer the reader to $[28,29,35-37,54,57,58,63]$ for some recent related contributions in this area.

Finally, we briefly summarize the basic notation used in this paper: Let $\mathcal{G}, \mathcal{H}, \mathfrak{H}$, etc., be separable complex Hilbert spaces, $(\cdot, \cdot)_{\mathcal{H}}$ the scalar product in $\mathcal{H}$ (linear in the first factor), and $I_{\mathcal{H}}$ the identity operator in $\mathcal{H}$. If $T$ is a linear operator mapping (a subspace of) a Hilbert space into another, $\operatorname{dom}(T)$ denotes the domain and $\operatorname{ran}(T)$ is the range of $T$. The closure of a closable operator $S$ is denoted by $\bar{S}$. The spectrum and resolvent set of a closed linear operator in $\mathcal{H}$ will be denoted by $\sigma(\cdot)$ and $\rho(\cdot)$, respectively. The Banach space of bounded linear operators in $\mathcal{H}$ is denoted by $\mathcal{L}(\mathcal{H})$; in the context of two Hilbert spaces, $\mathcal{H}_{j}, j=1,2$, we use the analogous abbreviation $\mathcal{L}\left(\mathcal{H}_{1}, \mathcal{H}_{2}\right)$. The $p$-th Schatten-von Neumann ideal consists of compact operators with singular values in $l^{p}, p>0$, and is denoted by $\mathfrak{S}_{p}(\mathcal{H})$ and $\mathfrak{S}_{p}\left(\mathcal{H}_{1}, \mathcal{H}_{2}\right)$. For $\Omega \subseteq \mathbb{R}^{n}$ nonempty, $n \in \mathbb{N}$, we suppress the $n$-dimensional Lebesgue measure $d^{n} x$ and use the shorthand notation $L^{2}(\Omega):=L^{2}\left(\Omega ; d^{n} x\right)$; similarly, if $\partial \Omega$ is sufficiently regular we write $L^{2}(\partial \Omega):=L^{2}\left(\partial \Omega ; d^{n-1} \sigma\right)$, with $d^{n-1} \sigma$ the surface measure on $\partial \Omega$. We also abbreviate $\mathbb{C}_{ \pm}:=\{z \in \mathbb{C} \mid \operatorname{Im}(z) \gtrless 0\}$ and $\mathbb{N}_{0}=\mathbb{N} \cup\{0\}$.

\section{Quasi boundary triples and their Weyl functions}

In this section we recall the concept of quasi boundary triples and their Weyl functions from extension theory of symmetric operators. We shall make use of these notions in Sect. 4 and formulate our main abstract result Theorem 4.1 in terms of the Weyl function of a quasi boundary triple. In Sects. 5 and 6 quasi boundary triples and their Weyl functions are used to parametrize self-adjoint Schrödinger operators and selfadjoint elliptic differential operators with suitable boundary conditions. We refer to $[2,3]$ for more details on quasi boundary triples and to [4-6,8] for some applications; for the related notions of generalized and ordinary boundary triples see [18,21-23,32, 69].

Throughout this section let $\mathfrak{H}$ be a separable Hilbert space and let $S$ be a densely defined closed symmetric operator in $\mathfrak{H}$.

Definition 2.1 Let $T \subset S^{*}$ be a linear operator in $\mathfrak{H}$ such that $\bar{T}=S^{*}$. A triple $\left\{\mathcal{G}, \Gamma_{0}, \Gamma_{1}\right\}$ is said to be a quasi boundary triple for $T \subset S^{*}$ if $\mathcal{G}$ is a Hilbert space and $\Gamma_{0}, \Gamma_{1}: \operatorname{dom}(T) \rightarrow \mathcal{G}$ are linear mappings such that the following conditions (i)-(iii) are satisfied:

(i) The abstract Green's identity

$$
(T f, g)_{\mathfrak{H}}-(f, T g)_{\mathfrak{H}}=\left(\Gamma_{1} f, \Gamma_{0} g\right)_{\mathcal{G}}-\left(\Gamma_{0} f, \Gamma_{1} g\right)_{\mathcal{G}}
$$

holds for all $f, g \in \operatorname{dom}(T)$.

(ii) The range of the map $\left(\Gamma_{0}, \Gamma_{1}\right)^{\top}: \operatorname{dom}(T) \rightarrow \mathcal{G} \times \mathcal{G}$ is dense.

(iii) The operator $A_{0}:=T \uparrow \operatorname{ker}\left(\Gamma_{0}\right)$ is self-adjoint in $\mathfrak{H}$. 
The next theorem from [2,3] is useful in the applications in Sects. 5 and 6; it contains a sufficient condition for a triple $\left\{\mathcal{G}, \Gamma_{0}, \Gamma_{1}\right\}$ to be a quasi boundary triple.

Theorem 2.2 Let $\mathfrak{H}$ and $\mathcal{G}$ be separable Hilbert spaces and let $T$ be a linear operator in $\mathfrak{H}$. Assume that $\Gamma_{0}, \Gamma_{1}: \operatorname{dom}(T) \rightarrow \mathcal{G}$ are linear mappings such that the following conditions (i)-(iii) hold:

(i) The abstract Green's identity

$$
(T f, g)_{\mathfrak{H}}-(f, T g)_{\mathfrak{H}}=\left(\Gamma_{1} f, \Gamma_{0} g\right)_{\mathcal{G}}-\left(\Gamma_{0} f, \Gamma_{1} g\right)_{\mathcal{G}}
$$

holds for all $f, g \in \operatorname{dom}(T)$.

(ii) The range of $\left(\Gamma_{0}, \Gamma_{1}\right)^{\top}: \operatorname{dom}(T) \rightarrow \mathcal{G} \times \mathcal{G}$ is dense and $\operatorname{ker}\left(\Gamma_{0}\right) \cap \operatorname{ker}\left(\Gamma_{1}\right)$ is dense in $\mathfrak{H}$.

(iii) $T \uparrow \operatorname{ker}\left(\Gamma_{0}\right)$ is an extension of a self-adjoint operator $A_{0}$.

Then

$$
S:=T \uparrow\left(\operatorname{ker}\left(\Gamma_{0}\right) \cap \operatorname{ker}\left(\Gamma_{1}\right)\right)
$$

is a densely defined closed symmetric operator in $\mathfrak{H}$ such that $\bar{T}=S^{*}$ holds and the triple $\left\{\mathcal{G}, \Gamma_{0}, \Gamma_{1}\right\}$ is a quasi boundary triple for $S^{*}$ with $A_{0}=T \uparrow \operatorname{ker}\left(\Gamma_{0}\right)$.

Next, we recall the definition of the $\gamma$-field $\gamma$ and Weyl function $M$ associated to a quasi boundary triple, which is formally the same as in [22,23] for the case of ordinary or generalized boundary triples. Let $\left\{\mathcal{G}, \Gamma_{0}, \Gamma_{1}\right\}$ be a quasi boundary triple for $T \subset S^{*}$ with $A_{0}=T \uparrow \operatorname{ker}\left(\Gamma_{0}\right)$ and note that the direct sum decomposition

$$
\operatorname{dom}(T)=\operatorname{dom}\left(A_{0}\right) \dot{+} \operatorname{ker}\left(T-z I_{\mathfrak{H}}\right)=\operatorname{ker}\left(\Gamma_{0}\right) \dot{+} \operatorname{ker}\left(T-z I_{\mathfrak{H}}\right)
$$

of $\operatorname{dom}(T)$ holds for all $z \in \rho\left(A_{0}\right)$. Hence the mapping $\Gamma_{0}\left\lceil\operatorname{ker}\left(T-z I_{\mathfrak{H}}\right)\right.$ is injective for all $z \in \rho\left(A_{0}\right)$ and its range coincides with $\operatorname{ran}\left(\Gamma_{0}\right)$.

Definition 2.3 Let $T \subset S^{*}$ be a linear operator in $\mathfrak{H}$ such that $\bar{T}=S^{*}$ and let $\left\{\mathcal{G}, \Gamma_{0}, \Gamma_{1}\right\}$ be a quasi boundary triple for $T \subset S^{*}$ with $A_{0}=T \uparrow \operatorname{ker}\left(\Gamma_{0}\right)$. The $\gamma$-field $\gamma$ and the Weyl function $M$ corresponding to $\left\{\mathcal{G}, \Gamma_{0}, \Gamma_{1}\right\}$ are operator-valued functions on $\rho\left(A_{0}\right)$ which are defined by

$z \mapsto \gamma(z):=\left(\Gamma_{0}\left\lceil\operatorname{ker}\left(T-z I_{\mathfrak{H}}\right)\right)^{-1}\right.$ and $z \mapsto M(z):=\Gamma_{1}\left(\Gamma_{0}\left\lceil\operatorname{ker}\left(T-z I_{\mathfrak{H}}\right)\right)^{-1}\right.$.

Various properties of the $\gamma$-field and Weyl function were provided in [2,3], see also $[18,21-23,69]$ for the special cases of ordinary and generalized boundary triples. We briefly review some items which are important for our purposes. Note first that the values $\gamma(z), z \in \rho\left(A_{0}\right)$, of the $\gamma$-field are operators defined on the dense subspace $\operatorname{ran}\left(\Gamma_{0}\right) \subset \mathcal{G}$ which map onto $\operatorname{ker}\left(T-z I_{\mathfrak{H}}\right) \subset \mathfrak{H}$. The operators $\gamma(z), z \in \rho\left(A_{0}\right)$, are bounded and admit continuous extensions $\overline{\gamma(z)} \in \mathcal{L}(\mathcal{G}, \mathfrak{H})$. For the adjoint operators $\gamma(z)^{*} \in \mathcal{L}(\mathfrak{H}, \mathcal{G}), z \in \rho\left(A_{0}\right)$, it follows that

$$
\gamma(z)^{*}=\Gamma_{1}\left(A_{0}-\bar{z} I_{\mathfrak{H}}\right)^{-1}, \quad z \in \rho\left(A_{0}\right),
$$


and, in particular, $\operatorname{ran}\left(\gamma(z)^{*}\right)=\operatorname{ran}\left(\Gamma_{1}\left\lceil\operatorname{dom}\left(A_{0}\right)\right)\right.$ does not depend on $z \in \rho\left(A_{0}\right)$. It is also important to note that $\left(\operatorname{ran}\left(\gamma(z)^{*}\right)\right)^{\perp}=\operatorname{ker}(\gamma(z))=\{0\}$ and hence

$$
\overline{\operatorname{ran}\left(\gamma(z)^{*}\right)}=\mathcal{G}, \quad z \in \rho\left(A_{0}\right) .
$$

In the same way as for ordinary boundary triples one verifies

$$
\gamma(z) \varphi=\left(I_{\mathfrak{H}}+\left(z-z_{0}\right)\left(A_{0}-z I_{\mathfrak{H}}\right)^{-1}\right) \gamma\left(z_{0}\right) \varphi, \quad z, z_{0} \in \rho\left(A_{0}\right), \quad \varphi \in \operatorname{ran}\left(\Gamma_{0}\right),
$$

and therefore $z \mapsto \gamma(z) \varphi$ is holomorphic on $\rho\left(A_{0}\right)$ for all $\varphi \in \operatorname{ran}\left(\Gamma_{0}\right)$. The relation (2.4) extends by continuity to

$$
\overline{\gamma(z)}=\left(I_{\mathfrak{H}}+\left(z-z_{0}\right)\left(A_{0}-z I_{\mathfrak{H}}\right)^{-1}\right) \overline{\gamma\left(z_{0}\right)} \in \mathcal{L}(\mathcal{G}, \mathfrak{H}), \quad z, z_{0} \in \rho\left(A_{0}\right),
$$

and it follows that $z \mapsto \overline{\gamma(z)}$ is a holomorphic $\mathcal{L}(\mathcal{G}, \mathfrak{H})$-valued operator function. According to [6, Lemma 2.4] the identities

$$
\frac{d^{k}}{d z^{k}} \overline{\gamma(z)}=k !\left(A_{0}-z I_{\mathfrak{H}}\right)^{-k} \overline{\gamma(z)}, \quad \frac{d^{k}}{d z^{k}} \gamma(\bar{z})^{*}=k ! \gamma(\bar{z})^{*}\left(A_{0}-z I_{\mathfrak{H}}\right)^{-k},
$$

hold for all $k \in \mathbb{N}_{0}$ and $z \in \rho\left(A_{0}\right)$.

The values $M(z), z \in \rho\left(A_{0}\right)$, of the Weyl function $M$ associated to a quasi boundary triple are operators in $\mathcal{G}$ and it follows from Definition 2.3 that

$$
\operatorname{dom}(M(z))=\operatorname{ran}\left(\Gamma_{0}\right) \text { and } \operatorname{ran}(M(z)) \subset \operatorname{ran}\left(\Gamma_{1}\right)
$$

hold for all $z \in \rho\left(A_{0}\right)$. In particular, the operators $M(z), z \in \rho\left(A_{0}\right)$, are densely defined in $\mathcal{G}$. With the help of the abstract Green's identity one concludes that for $z, z_{0} \in \rho\left(A_{0}\right)$ and $\varphi, \psi \in \operatorname{ran}\left(\Gamma_{0}\right)$ the Weyl function and the $\gamma$-field satisfy

$$
(M(z) \varphi, \psi)_{\mathcal{G}}-\left(\varphi, M\left(z_{0}\right) \psi\right)_{\mathcal{G}}=\left(z-\overline{z_{0}}\right)\left(\gamma(z) \varphi, \gamma\left(z_{0}\right) \psi\right)_{\mathcal{G}}
$$

and hence $M(z) \subset M(\bar{z})^{*}$ and the operators $M(z)$ are closable for all $z \in \rho\left(A_{0}\right)$. From (2.7) it also follows that the Weyl function and the $\gamma$-field are connected via

$$
M(z) \varphi-M\left(z_{0}\right)^{*} \varphi=\left(z-\overline{z_{0}}\right) \gamma\left(z_{0}\right)^{*} \gamma(z) \varphi, \quad z, z_{0} \in \rho\left(A_{0}\right), \quad \varphi \in \operatorname{ran}\left(\Gamma_{0}\right) .
$$

From (2.8) and (2.4) one obtains

$$
\operatorname{Im}(M(z)) \varphi=\operatorname{Im}(z) \gamma(z)^{*} \gamma(z) \varphi, \quad z \in \rho\left(A_{0}\right), \quad \varphi \in \operatorname{ran}\left(\Gamma_{0}\right),
$$

and

$$
\begin{aligned}
M(z) \varphi= & \operatorname{Re}\left(M\left(z_{0}\right)\right) \varphi \\
& +\gamma\left(z_{0}\right)^{*}\left(\left(z-\operatorname{Re}\left(z_{0}\right)\right)+\left(z-z_{0}\right)\left(z-\overline{z_{0}}\right)\left(A_{0}-z I_{\mathfrak{H}}\right)^{-1}\right) \gamma\left(z_{0}\right) \varphi
\end{aligned}
$$


for all $z, z_{0} \in \rho\left(A_{0}\right)$ and $\varphi \in \operatorname{ran}\left(\Gamma_{0}\right)$. One observes that $z \mapsto M(z) \varphi$ is holomorphic on $\rho\left(A_{0}\right)$ for all $\varphi \in \operatorname{ran}\left(\Gamma_{0}\right)$ and by (2.9) the imaginary part of $M(z)$ is a bounded operator in $\mathcal{G}$ which admits a bounded continuation to

$$
\overline{\operatorname{Im}(M(z))}=\operatorname{Im}(z) \gamma(z)^{*} \overline{\gamma(z)} \in \mathcal{L}(\mathcal{G})
$$

Furthermore, the derivatives $\frac{d^{k}}{d z^{k}} M(z), k \in \mathbb{N}$, of the Weyl function are densely defined bounded operators in $\mathcal{G}$ and according to [6, Lemma 2.4] one has

$$
\overline{\frac{d^{k}}{d z^{k}} M(z)}=k ! \gamma(\bar{z})^{*}\left(A_{0}-z I_{\mathfrak{H}}\right)^{-(k-1)} \overline{\gamma(z)}, \quad k \in \mathbb{N}, \quad z \in \rho\left(A_{0}\right) .
$$

If the values $M(z)$ are densely defined bounded operators for some, and hence for all $z \in \rho\left(A_{0}\right)$ then

$$
\frac{d^{k}}{d z^{k}} \overline{M(z)}=k ! \gamma(\bar{z})^{*}\left(A_{0}-z I_{\mathfrak{H}}\right)^{-(k-1)} \overline{\gamma(z)}, \quad k \in \mathbb{N}, \quad z \in \rho\left(A_{0}\right) .
$$

The next result will be used in the formulation and proof of our abstract representation formula for the spectral shift function in Sect. 4. The existence of a quasi boundary triple follows from [8, Proposition 2.9(i)] and the Krein-type resolvent formula in (2.14) is a special case of [3, Corollary 6.17] or [5, Corollary 3.9].

Proposition 2.4 Let $A$ and $B$ be self-adjoint operators in $\mathfrak{H}$ and assume that the closed symmetric operator $S=A \cap B$ is densely defined. Then the closure of the operator

$$
T=S^{*} \uparrow(\operatorname{dom}(A)+\operatorname{dom}(B))
$$

coincides with $S^{*}$ and there exists a quasi boundary triple $\left\{\mathcal{G}, \Gamma_{0}, \Gamma_{1}\right\}$ for $T \subset S^{*}$ such that

$$
A=T \uparrow \operatorname{ker}\left(\Gamma_{0}\right) \quad \text { and } \quad B=T \uparrow \operatorname{ker}\left(\Gamma_{1}\right) .
$$

Furthermore, if $\gamma$ and $M$ are the corresponding $\gamma$-field and Weyl function then

$$
\left(B-z I_{\mathfrak{H}}\right)^{-1}-\left(A-z I_{\mathfrak{H}}\right)^{-1}=-\gamma(z) M(z)^{-1} \gamma(\bar{z})^{*}, \quad z \in \rho(A) \cap \rho(B) .
$$

\section{Logarithms of operator-valued Nevanlinna functions}

In this section we study the logarithm of operator-valued Nevanlinna (or NevanlinnaHerglotz, resp., Riesz-Herglotz) functions. Here we shall recall some of the results formulated in [26, Section 2] which go back to [10,60-62], and slightly extend and reformulate these in a form convenient for our subsequent purposes.

We first recall the integral representation of the logarithm that corresponds to the cut along the negative imaginary axis,

$$
\log (z)=-i \int_{0}^{\infty}\left(\frac{1}{z+i \lambda}-\frac{1}{1+i \lambda}\right) d \lambda, \quad z \in \mathbb{C}, \quad z \neq-i \lambda, \quad \lambda \geq 0 .
$$


Next, let $\mathcal{G}$ be a separable Hilbert space and let $K \in \mathcal{L}(\mathcal{G})$ be a bounded operator such that $\operatorname{Im}(K) \geq 0$ and $0 \subset \rho(K)$. We use

$$
\log (K):=-i \int_{0}^{\infty}\left[\left(K+i \lambda I_{\mathcal{G}}\right)^{-1}-(1+i \lambda)^{-1} I_{\mathcal{G}}\right] d \lambda
$$

as the definition of the logarithm of the operator $K$. Then $\log (K) \in \mathcal{L}(\mathcal{G})$ by [26, Lemma 2.6] and in the special case that $K \in \mathcal{L}(\mathcal{G})$ is self-adjoint and $0 \in \rho(K)$, it follows from [26, Lemma 2.7] that

$$
\operatorname{Im}(\log (K))=\pi E_{K}((-\infty, 0)),
$$

where $E_{K}(\cdot)$ is the spectral measure of $K$. In particular, if $K \in \mathcal{L}(\mathcal{G})$ is self-adjoint and $0 \in \rho(K)$ then $\sigma(K) \subset(0, \infty)$ if and only if $\log (K)$ is a self-adjoint operator.

In the next lemma we show that besides $\log (K)$ also $\log \left(K^{*}\right)$ is well-defined via (3.2) when $K$ is a dissipative operator with spectrum off the imaginary axis (cf. [26, Lemmas 2.6, 2.7]).

Lemma 3.1 Let $K \in \mathcal{L}(\mathcal{G})$ be a dissipative operator such that $i \lambda \in \rho(K)$ for all $\lambda \geq 0$, and define

$$
\log \left(K^{*}\right):=-i \int_{0}^{\infty}\left[\left(K^{*}+i \lambda I_{\mathcal{G}}\right)^{-1}-(1+i \lambda)^{-1} I_{\mathcal{G}}\right] d \lambda .
$$

Then $\log \left(K^{*}\right) \in \mathcal{L}(\mathcal{G})$.

Proof From $\sigma\left(K^{*}\right)=\{z \in \mathbb{C} \mid \bar{z} \in \sigma(K)\}$ and the assumption $i \lambda \in \rho(K)$ for $\lambda \geq 0$ it is clear that $-i \lambda \in \rho\left(K^{*}\right)$ for $\lambda \geq 0$. Since $K$ is dissipative it follows that $K^{*}$ is accretive, that is, $\operatorname{Im}\left(K^{*}\right) \leq 0$. For $\delta>0$ one estimates

$$
\begin{aligned}
\left\|\log \left(K^{*}\right)\right\|_{\mathcal{L}(\mathcal{G})} \leq & \int_{0}^{\delta}\left[\left\|\left(K^{*}+i \lambda I_{\mathcal{G}}\right)^{-1}\right\|_{\mathcal{L}(\mathcal{G})}+1\right] d \lambda \\
& +\int_{\delta}^{\infty}\left\|\left(K^{*}+i \lambda I_{\mathcal{G}}\right)^{-1}\right\|_{\mathcal{L}(\mathcal{G})}\left(\|K\|_{\mathcal{L}(\mathcal{G})}+1\right) \lambda^{-1} d \lambda .
\end{aligned}
$$

For $0<\lambda<\left\|\left(K^{*}\right)^{-1}\right\|_{\mathcal{L}(\mathcal{G})}^{-1}$ one has

$$
\left\|\left(K^{*}+i \lambda I_{\mathcal{G}}\right)^{-1}\right\|_{\mathcal{L}(\mathcal{G})} \leq \frac{\left\|\left(K^{*}\right)^{-1}\right\|_{\mathcal{L}(\mathcal{G})}}{1-\lambda\left\|\left(K^{*}\right)^{-1}\right\|_{\mathcal{L}(\mathcal{G})}},
$$

and with the choice $\delta=\left(2\left\|\left(K^{*}\right)^{-1}\right\|_{\mathcal{L}(\mathcal{G})}\right)^{-1}$ it follows that the first integral in (3.5) is bounded. In order to show that the second integral in (3.5) is also bounded it suffices to show that

$$
\left\|\left(K^{*}+i \lambda I_{\mathcal{G}}\right)^{-1}\right\|_{\mathcal{L}(\mathcal{G})} \leq \frac{1}{\lambda-\left\|K^{*}\right\|_{\mathcal{L}(\mathcal{G})}}, \quad \lambda>\left\|K^{*}\right\|_{\mathcal{L}(\mathcal{G})} .
$$


In fact, since $\operatorname{Im}\left(K^{*}+i\left\|K^{*}\right\|_{\mathcal{L}(\mathcal{G})} I_{\mathcal{G}}\right) \geq 0$ one estimates for $\lambda>\left\|K^{*}\right\|_{\mathcal{L}(\mathcal{G})}$,

$$
\begin{aligned}
0 & \leq\left(\lambda-\left\|K^{*}\right\|_{\mathcal{L}(\mathcal{G})}\right)\|f\|_{\mathcal{G}}^{2}=\operatorname{Im}\left(i\left(\lambda-\left\|K^{*}\right\|_{\mathcal{L}(\mathcal{G})}\right) f, f\right)_{\mathcal{G}} \\
& \leq \operatorname{Im}\left(\left(i \lambda-i\left\|K^{*}\right\|_{\mathcal{L}(\mathcal{G})}\right) f, f\right)_{\mathcal{G}}+\operatorname{Im}\left(\left(K^{*}+i\left\|K^{*}\right\|_{\mathcal{L}(\mathcal{G})} I_{\mathcal{G}}\right) f, f\right)_{\mathcal{G}} \\
& =\operatorname{Im}\left(\left(K^{*}+i \lambda I_{\mathcal{G}}\right) f, f\right)_{\mathcal{G}} \leq\left\|\left(K^{*}+i \lambda I_{\mathcal{G}}\right) f\right\|_{\mathcal{G}}\|f\|_{\mathcal{G}}
\end{aligned}
$$

and for $f \neq 0$ this yields

$$
0 \leq\left(\lambda-\left\|K^{*}\right\|_{\mathcal{L}(\mathcal{G})}\right)\|f\|_{\mathcal{G}} \leq\left\|\left(K^{*}+i \lambda I_{\mathcal{G}}\right) f\right\|_{\mathcal{G}}
$$

Since $-i \lambda \in \rho\left(K^{*}\right)$ there exists $g \in \mathcal{G}$ such that $f=\left(K^{*}+i \lambda I_{\mathcal{G}}\right)^{-1} g$ and then (3.8) has the form

$$
\left\|\left(K^{*}+i \lambda I_{\mathcal{G}}\right)^{-1} g\right\|_{\mathcal{G}} \leq \frac{1}{\lambda-\left\|K^{*}\right\|_{\mathcal{L}(\mathcal{G})}}\|g\|_{\mathcal{G}}, \quad \lambda>\left\|K^{*}\right\|_{\mathcal{L}(\mathcal{G})}
$$

This implies (3.6), and hence the second integral in the estimate (3.5) is finite. Thus, $\log \left(K^{*}\right)$ in (3.4) is a bounded operator in $\mathcal{G}$.

We recall that a function $N: \mathbb{C}_{+} \rightarrow \mathcal{L}(\mathcal{G})$ is an operator-valued Nevanlinna (or Riesz-Herglotz) function if $N$ is holomorphic and $\operatorname{Im}(N(z)) \geq 0$ holds for all $z \in \mathbb{C}_{+}$. An $\mathcal{L}(\mathcal{G})$-valued Nevanlinna function is extended onto $\mathbb{C}_{-}$by setting

$$
N(z):=N(\bar{z})^{*}, \quad z \in \mathbb{C}_{-} .
$$

We shall say that a Nevanlinna function $N$ admits an analytic continuation by reflection with respect to some open subset $I \subset \mathbb{R}$ if $N$ can be continued analytically from $\mathbb{C}_{+}$ onto an open set $\mathcal{O} \subset \mathbb{C}$ which contains $I$ such that the values of the continuation in $\mathcal{O} \cap \mathbb{C}_{-}$coincide with the values of $N$ in (3.9) there.

Example 3.2 If $\sqrt{z}$ is fixed for $\mathbb{C} \backslash[0, \infty)$ by $\operatorname{Im}(\sqrt{z})>0$ and by $\sqrt{z} \geq 0$ for $z \in$ $[0, \infty)$ then $\mathbb{C}_{+} \ni z \mapsto \sqrt{z}$ is a (scalar) Nevanlinna function which admits an analytic continuation by reflection with respect to $(-\infty, 0)$, but it does not admit an analytic continuation by reflection with respect to any open subinterval of $[0, \infty)$.

An operator-valued Nevanlinna function admits a minimal operator representation via the resolvent of a self-adjoint operator or relation in an auxiliary or larger Hilbert space (see, e.g., $[10,38,50,60])$. More precisely, if $N: \mathbb{C}_{+} \rightarrow \mathcal{L}(\mathcal{G})$ is a Nevanlinna function and $z_{0} \in \mathbb{C}_{+}$is fixed then there exists a Hilbert space $\mathcal{K}$, a self-adjoint operator or self-adjoint relation $L$ in $\mathcal{K}$ and an operator $R \in \mathcal{L}(\mathcal{G}, \mathcal{K})$ (depending on the choice of $z_{0}$ ) such that

$$
N(z)=\operatorname{Re}\left(N\left(z_{0}\right)\right)+\left(z-\operatorname{Re}\left(z_{0}\right)\right) R^{*} R+\left(z-z_{0}\right)\left(z-\bar{z}_{0}\right) R^{*}\left(L-z I_{\mathcal{K}}\right)^{-1} R
$$

holds for $z \in \mathbb{C}_{+}$. If $N$ satisfies the condition

$$
\lim _{y \uparrow+\infty} y^{-1}(N(i y) h, h)_{\mathcal{G}}=0 \text { for all } h \in \mathcal{G},
$$


then $L$ in (3.10) is a self-adjoint operator in $\mathcal{K}$; cf. [50, Corollary 2.5]. The representation (3.10) also holds for $z \in \mathbb{C}_{-}$when $N$ is extended onto $\mathbb{C}_{-}$via (3.9). Note that the model can be chosen minimal, that is, the minimality condition

$$
\mathcal{K}=\operatorname{clsp}\left\{\left(I_{\mathcal{K}}+\left(z-z_{0}\right)\left(L-z I_{\mathcal{K}}\right)^{-1}\right) R h \mid z \in \mathbb{C} \backslash \mathbb{R}, h \in \mathcal{G}\right\}
$$

is satisfied, in which case the resolvent set $\rho(L)$ of $L$ coincides with the maximal domain of analyticity of the function $N$. In particular, in this case $N$ admits an analytic continuation by reflection with respect to an open subset $I \subset \mathbb{R}$ if and only if $I \subset \rho(L)$, and the open subset $\rho(L) \cap \mathbb{R}$ is maximal with this property.

Next, assume that $N$ is an $\mathcal{L}(\mathcal{G})$-valued Nevanlinna function and suppose that $N(z)^{-1} \in \mathcal{L}(\mathcal{G})$ for some, and hence (by [26, Lemma 2.3]) for all $z \in \mathbb{C} \backslash \mathbb{R}$. Then we define for $z \in \mathbb{C}_{+}$the $\operatorname{logarithm} \log (N(z))$ in accordance with (3.2) by

$$
\log (N(z)):=-i \int_{0}^{\infty}\left[\left(N(z)+i \lambda I_{\mathcal{G}}\right)^{-1}-(1+i \lambda)^{-1} I_{\mathcal{G}}\right] d \lambda
$$

and extend the function $\log (N)$ onto $\mathbb{C}_{-}$by reflection,

$$
\log (N(z)):=(\log (N(\bar{z})))^{*}, \quad z \in \mathbb{C}_{-}
$$

(cf. (3.9)). By [26, Lemma 2.8] the function $z \mapsto \log (N(z))$ is also an $\mathcal{L}(\mathcal{G})$-valued Nevanlinna function and satisfies

$$
0 \leq \operatorname{Im}(\log (N(z))) \leq \pi I_{\mathcal{G}}, \quad z \in \mathbb{C}_{+}
$$

The following theorem is a variant and slight extension of [26, Theorem 2.10], the new and important feature here is that we provide a sufficient condition in terms of the function $N$ such that $\log (N)$ admits an analytic continuation by reflection with respect to some real interval and a corresponding integral representation there.

Theorem 3.3 Let $N: \mathbb{C} \backslash \mathbb{R} \rightarrow \mathcal{L}(\mathcal{G})$ be a Nevanlinna function and assume that $N(z)^{-1} \in \mathcal{L}(\mathcal{G})$ for some, and hence for all $z \in \mathbb{C} \backslash \mathbb{R}$. Then there exists a weakly Lebesgue measurable operator-valued function $\lambda \mapsto \Xi(\lambda) \in \mathcal{L}(\mathcal{G})$ on $\mathbb{R}$ such that

$$
\Xi(\lambda)=\Xi(\lambda)^{*} \text { and } 0 \leq \Xi(\lambda) \leq I_{\mathcal{G}} \text { for a.e. } \lambda \in \mathbb{R} \text {, }
$$

and the Nevanlinna function $\log (N): \mathbb{C} \backslash \mathbb{R} \rightarrow \mathcal{L}(\mathcal{G})$ in (3.12)-(3.13) admits an integral representation of the form

$$
\log (N(z))=C+\int_{\mathbb{R}}\left(\frac{1}{\lambda-z}-\frac{\lambda}{1+\lambda^{2}}\right) \Xi(\lambda) d \lambda,
$$

where $C=\operatorname{Re}(\log (N(i))) \in \mathcal{L}(\mathcal{G})$ is a self-adjoint operator and the integral is understood in the weak sense.

If, in addition, $N$ admits an analytic continuation by reflection with respect to an open interval $I \subset \mathbb{R}$ such that $\sigma(N(z)) \subset(\varepsilon, \infty)$ for some $\varepsilon>0$ and all $z \in I$, then 
also $\log (N)$ admits an analytic continuation by reflection with respect to $I, \Xi(\lambda)=0$ for a.e. $\lambda \in I$, and (3.16) remains valid for $z \in I$.

Proof We make use of the representation (3.10) applied to the Nevanlinna function $\log (N)$ with $z_{0}=i$. Then there exists a Hilbert space $\mathcal{K}$ and $R \in \mathcal{L}(\mathcal{G}, \mathcal{K})$ such that

$$
\log (N(z))=C+z R^{*} R+\left(1+z^{2}\right) R^{*}\left(L-z I_{\mathcal{K}}\right)^{-1} R, \quad z \in \mathbb{C} \backslash \mathbb{R},
$$

where $C=\operatorname{Re}(\log (N(i))) \in \mathcal{L}(\mathcal{G})$ is a self-adjoint operator. For $h \in \mathcal{G}$ it follows from (3.17) and (3.14) that

$$
\left.\left.\lim _{y \rightarrow+\infty} \frac{1}{y} \operatorname{Re}\left(\log ((N(i y)) h, h)_{\mathcal{G}}\right)\right)=0=\lim _{y \rightarrow+\infty} \frac{1}{y} \operatorname{Im}\left(\log ((N(i y)) h, h)_{\mathcal{G}}\right)\right),
$$

so that (3.11) holds for the function $\log (N)$. Hence $L$ in (3.17) is a self-adjoint operator in $\mathcal{K}$ (cf. [26, Lemma 2.9]). We can assume that the model is chosen minimal and hence $\rho(L)$ coincides with the maximal domain of analyticity of the Nevanlinna function $\log (N)$.

In order to prove (3.15) and (3.16) one can argue in the same way as in the proof of [26, Theorem 2.10]. Let $\lambda \mapsto E_{L}(\lambda)$ be the spectral function of $L$ such that $\lim _{\lambda \downarrow-\infty}\left(E_{L}(\lambda) h, h\right)_{\mathcal{G}}=0$. Then (3.17) yields

$$
(\log (N(z)) h, h)_{\mathcal{G}}=(C h, h)_{\mathcal{G}}+\int_{\mathbb{R}}\left(\frac{1}{\lambda-z}-\frac{\lambda}{1+\lambda^{2}}\right)\left(1+\lambda^{2}\right) d\left(R^{*} E_{L}(\lambda) R h, h\right)_{\mathcal{G}}
$$

for $h \in \mathcal{G}, z \in \mathbb{C} \backslash \mathbb{R}$, and (3.14) and the Stieltjes inversion formula imply that the measures

$$
d \omega_{h}(\cdot)=\left(1+\lambda^{2}\right) d\left(R^{*} E_{L}(\cdot) R h, h\right)_{\mathcal{G}}
$$

are absolutely continuous with respect to the Lebesgue measure $d \lambda$ and there exist measurable functions $\xi_{h}$ with $0 \leq \xi_{h}(\lambda) \leq\|h\|_{\mathcal{G}}^{2}$ for a.e. $\lambda \in \mathbb{R}$ such that $d \omega_{h}(\lambda)=$ $\xi_{h}(\lambda) d \lambda$. Hence there exists a weakly Lebesgue measurable function $\lambda \mapsto \Xi(\lambda)$ such that

$$
\xi_{h}(\lambda)=(\Xi(\lambda) h, h)_{\mathcal{G}} \text { and } 0 \leq \Xi(\lambda) \leq I_{\mathcal{G}},
$$

proving (3.15) and (3.16).

Next, assume that $N$ admits an analytic continuation by reflection with respect to an open interval $I \subset \mathbb{R}$ such that $\sigma(N(z)) \subset(\varepsilon, \infty)$ for some $\varepsilon>0$ and all $z \in I$. Fix some $z_{0} \in I$ and an open ball $\mathcal{B}_{z_{0}} \subset \mathbb{C}$ centered at $z_{0}$ such that $N$ is analytic on $\mathcal{B}_{z_{0}}$. Since $\sigma\left(N\left(z_{0}\right)\right) \subset(\varepsilon, \infty)$ we can assume that $\mathcal{B}_{z_{0}}$ was chosen such that

$$
\sigma(N(z)) \subset\{z \in \mathbb{C} \mid \varepsilon / 2<\operatorname{Re}(z), 0 \leq \operatorname{Im}(z)<\varepsilon\}, \quad z \in \mathcal{B}_{z_{0}} \cap \mathbb{C}_{+},
$$

and hence the operators $N(z), z \in \mathcal{B}_{z_{0}} \cap \mathbb{C}_{+}$, satisfy the assumptions in Lemma 3.1. Therefore, the operators

$$
\log \left(N(z)^{*}\right):=-i \int_{0}^{\infty}\left[\left(N(z)^{*}+i \lambda I_{\mathcal{G}}\right)^{-1}-(1+i \lambda)^{-1} I_{\mathcal{G}}\right] d \lambda, \quad z \in \mathcal{B}_{z_{0}} \cap \mathbb{C}_{+},
$$


are well-defined, and since $N(\bar{z})=N(z)^{*}$, it follows that

$$
\log (N(z))=-i \int_{0}^{\infty}\left[\left(N(z)+i \lambda I_{\mathcal{G}}\right)^{-1}-(1+i \lambda)^{-1} I_{\mathcal{G}}\right] d \lambda, \quad z \in \mathcal{B}_{z_{0}} \cap \mathbb{C}_{-},
$$

are well-defined, bounded operators in $\mathcal{G}$. Furthermore, Lemma 3.1 also ensures that for $z \in \mathcal{B}_{z_{0}} \cap \mathbb{R}$ the operators

$$
\log (N(z)):=-i \int_{0}^{\infty}\left[\left(N(z)+i \lambda I_{\mathcal{G}}\right)^{-1}-(1+i \lambda)^{-1} I_{\mathcal{G}}\right] d \lambda, \quad z \in \mathcal{B}_{z_{0}} \cap \mathbb{R},
$$

are well-defined, bounded operators in $\mathcal{G}$. Thus for all $z \in \mathcal{B}_{z_{0}}$, the operators $\log (N(z))$ are well-defined via (3.12). It then follows from (3.12) that the function $z \mapsto \log (N(z))$ is analytic on $\mathcal{B}_{z_{0}}$ (cf. [26, Proof of Lemma 2.8]).

We shall now also make use of the logarithm

$$
\ln (z)=\int_{-\infty}^{0}\left(\frac{1}{\lambda-z}-\frac{\lambda}{1+\lambda^{2}}\right) d \lambda, \quad z \in \mathbb{C} \backslash(-\infty, 0],
$$

which corresponds to the cut along the negative real axis. Since

$$
\sigma(N(z)) \subset\{z \in \mathbb{C} \mid \varepsilon / 2<\operatorname{Re}(z),-\varepsilon<\operatorname{Im}(z)<\varepsilon\}, \quad z \in \mathcal{B}_{z_{0}},
$$

it follows that

$$
\ln (N(z))=\int_{-\infty}^{0}\left[\left(\lambda I_{\mathcal{G}}-N(z)\right)^{-1}-\lambda\left(1+\lambda^{2}\right)^{-1} I_{\mathcal{G}}\right] d \lambda, \quad z \in \mathcal{B}_{z_{0}},
$$

are well-defined operators and the function $z \mapsto \ln (N(z))$ is analytic on $\mathcal{B}_{z_{0}}$. In addition, (3.20) yields

$$
(\ln (N(z)))^{*}=\ln \left(N(z)^{*}\right), \quad z \in \mathcal{B}_{z_{0}} .
$$

As $\log (z)=\ln (z)($ see (3.1)) for all $z>0$ and $N(z)$ is self-adjoint for $z \in I$ it follows from the spectral theorem that

$$
\log (N(z))=\ln (N(z)), \quad z \in I,
$$

and hence $\log (N(z))=\ln (N(z)), z \in \mathcal{B}_{z_{0}}$, by analyticity. Therefore, (3.21) and $N(z)^{*}=N(\bar{z})$ yield

$$
(\log (N(z)))^{*}=(\ln (N(z)))^{*}=\ln \left(N(z)^{*}\right)=\ln (N(\bar{z}))=\log (N(\bar{z})), \quad z \in \mathcal{B}_{z_{0}} .
$$

It follows that $z \mapsto \log (N(z))$ is analytic on $\mathcal{B}_{z_{0}}$ and the continuation of $\log (N)$ onto $\mathcal{B}_{z_{0}} \cap \mathbb{C}_{-}$coincides with the extension of $\log (N)$ onto $\mathbb{C}_{-}$defined by

$$
\log (N(z))=(\log N(\bar{z}))^{*}, \quad z \in \mathbb{C}_{-},
$$


(cf. (3.9)). This reasoning applies to all $v \in I$ and hence we have shown that $\log (N)$ admits an analytic continuation by reflection with respect to $I$.

Since the operator model for $\log (N)$ is minimal the interval $I$ belongs to $\rho(L)$ and the representation (3.17) remains valid for $z \in \rho(L)$. It follows that the measures $d \omega_{h}(\cdot), h \in \mathcal{G}$, in (3.18) have no support in $I$ and hence their Radon-Nikodym deriatives satisfy $\xi_{h}(\lambda)=0$ for a.e. $\lambda \in I$. It follows that $(\Xi(\lambda) h, h)_{\mathcal{G}}=0$ for a.e. $\lambda \in I$ and all $h \in \mathcal{G}$. Since $\Xi(\lambda) \geq 0$ we conclude $\Xi(\lambda)=0$ for a.e. $\lambda \in I$.

In the next proposition we provide a sufficient condition such that the values of the function $\Xi$ are trace class operators and we express the traces of $\Xi(\lambda)$ in terms of certain weak limits of the imaginary part of $\log (N)$.

Proposition 3.4 Let $N: \mathbb{C} \backslash \mathbb{R} \rightarrow \mathcal{L}(\mathcal{G})$ be a Nevanlinna function such that $N(z)^{-1} \in$ $\mathcal{L}(\mathcal{G})$ for some, and hence for all $z \in \mathbb{C} \backslash \mathbb{R}$, and assume that $N$ admits an analytic continuation by reflection with respect to an open interval $I \subset \mathbb{R}$ such that $\sigma(N(\zeta)) \subset$ $(\varepsilon, \infty)$ for some $\varepsilon>0$ and all $\zeta \in I$. Consider

$$
\log (N(z))=C+\int_{\mathbb{R}}\left(\frac{1}{\lambda-z}-\frac{\lambda}{1+\lambda^{2}}\right) \Xi(\lambda) d \lambda,
$$

for $z \in(\mathbb{C} \backslash \mathbb{R}) \cup I$ with $\Xi(\lambda)=\Xi(\lambda)^{*}$ and $0 \leq \Xi(\lambda) \leq I_{\mathcal{G}}$ for a.e. $\lambda \in \mathbb{R}$ as in (3.15), and assume, in addition, that for some $k \in \mathbb{N}_{0}$ and some $\zeta \in I$,

$$
\frac{d^{2 k+1}}{d \zeta^{2 k+1}} \log (N(\zeta)) \in \mathfrak{S}_{1}(\mathcal{G})
$$

Then $0 \leq \Xi(\lambda) \in \mathfrak{S}_{1}(\mathcal{G})$ for a.e. $\lambda \in \mathbb{R}$, and

$$
\operatorname{tr}_{\mathcal{G}}(\Xi(\lambda))=\sum_{j \in J} \lim _{\varepsilon \downarrow 0} \frac{1}{\pi}\left(\operatorname{Im}(\log (N(\lambda+i \varepsilon))) \varphi_{j}, \varphi_{j}\right)_{\mathcal{G}}
$$

holds for any orthonormal basis $\left(\varphi_{j}\right)_{j \in J}$ in $\mathcal{G}(J \subseteq \mathbb{N}$ an appropriate index set $)$ and for a.e. $\lambda \in \mathbb{R}$. Furthermore, if (3.23) holds for some $\zeta \in I$ and $k=0$, that is,

$$
\frac{d}{d \zeta} \log (N(\zeta)) \in \mathfrak{S}_{1}(\mathcal{G})
$$

then $\operatorname{Im}(\log (N(z))) \in \mathfrak{S}_{1}(\mathcal{G})$ for all $z \in \mathbb{C} \backslash \mathbb{R}$, the limit

$$
\operatorname{Im}(\log (N(\lambda+i 0))):=\lim _{\varepsilon \downarrow 0} \operatorname{Im}(\log (N(\lambda+i \varepsilon))) \in \mathfrak{S}_{1}(\mathcal{G})
$$

exists for a.e. $\lambda \in \mathbb{R}$ in the norm of $\mathfrak{S}_{1}(\mathcal{G})$, and

$$
\operatorname{tr}_{\mathcal{G}}(\Xi(\lambda))=\frac{1}{\pi} \operatorname{tr}_{\mathcal{G}}(\operatorname{Im}(\log (N(\lambda+i 0)))) \quad \text { for a.e. } \lambda \in \mathbb{R}
$$


Proof The assumption (3.23) together with the integral representation (3.22) yields

$$
\begin{aligned}
& \frac{d^{2 k+1}}{d \zeta^{2 k+1}} \log (N(\zeta)) \\
& \quad=(2 k+1) ! \int_{\mathbb{R}} \frac{1}{(\lambda-\zeta)^{2 k+2}} \Xi(\lambda) d \lambda \in \mathfrak{S}_{1}(\mathcal{G}), \quad k \in \mathbb{N}_{0}, \quad \zeta \in I .
\end{aligned}
$$

Since $\Xi(\lambda) \geq 0$ by (3.15) and $(\lambda-\zeta)^{-2 k-2} \geq 0$ for all $\lambda \in \mathbb{R}, \zeta \in I$, it follows together with the assumption (3.23) that the integral in (3.27) is a nonnegative trace class operator. Similarly, as in [26, Proof of Theorem 2.10], the monotone convergence theorem yields $\Xi(\lambda) \in \mathfrak{S}_{1}(\mathcal{G})$ for a.e. $\lambda \in \mathbb{R}$. For $\varepsilon>0$ it follows from the integral representation (3.22) that

$$
(\operatorname{Im}(\log (N(\lambda+i \varepsilon))) h, h)_{\mathcal{G}}=\int_{\mathbb{R}} \frac{\varepsilon}{\left|\lambda^{\prime}-\lambda\right|^{2}+\varepsilon^{2}}\left(\Xi\left(\lambda^{\prime}\right) h, h\right)_{\mathcal{G}} d \lambda^{\prime}
$$

holds for all $h \in \mathcal{G}$ and all $\lambda \in \mathbb{R}$, and therefore the Stietljes inversion formula yields

$$
\lim _{\varepsilon \downarrow 0} \frac{1}{\pi}(\operatorname{Im}(\log (N(\lambda+i \varepsilon))) h, h)_{\mathcal{G}}=(\Xi(\lambda) h, h)_{\mathcal{G}} \quad \text { for a.e. } \lambda \in \mathbb{R} .
$$

Let $\left(\varphi_{j}\right)_{j \in J}$ be an orthonormal basis in $\mathcal{G}$. Then

$$
\lim _{\varepsilon \downarrow 0} \frac{1}{\pi}\left(\operatorname{Im}(\log (N(\lambda+i \varepsilon))) \varphi_{j}, \varphi_{j}\right)_{\mathcal{G}}=\left(\Xi(\lambda) \varphi_{j}, \varphi_{j}\right)_{\mathcal{G}}
$$

holds for all $\lambda \in \mathbb{R} \backslash \mathcal{A}_{j}$, where $\mathcal{A}_{j} \subset \mathbb{R}, j \in J$, is a set of Lebesgue measure zero. The countable union $\mathcal{A}:=\cup_{j \in J} \mathcal{A}_{j}$ is also a set of Lebesgue measure zero and for all $\lambda \in \mathbb{R} \backslash \mathcal{A}$ and all $\varphi_{j}$ one has (3.30). Taking into acount that $0 \leq \Xi(\lambda) \in \mathfrak{S}_{1}(\mathcal{G})$ for a.e. $\lambda \in \mathbb{R}$ this implies

$$
\sum_{j \in J} \lim _{\varepsilon \downarrow 0} \frac{1}{\pi}\left(\operatorname{Im}(\log (N(\lambda+i \varepsilon))) \varphi_{j}, \varphi_{j}\right)_{\mathcal{G}}=\sum_{j \in J}\left(\Xi(\lambda) \varphi_{j}, \varphi_{j}\right)_{\mathcal{G}}=\operatorname{tr}_{\mathcal{G}}(\Xi(\lambda))
$$

for a.e. $\lambda \in \mathbb{R}$, that is, (3.24) holds.

In the special case that (3.23) holds with $k=0$ the formula (3.27) has the form

$$
\frac{d}{d \zeta} \log (N(\zeta))=\int_{\mathbb{R}} \frac{1}{(\lambda-\zeta)^{2}} \Xi(\lambda) d \lambda \in \mathfrak{S}_{1}(\mathcal{G}), \quad \zeta \in I
$$

Since $0 \leq \Xi(\lambda) \in \mathfrak{S}_{1}(\mathcal{G})$ for a.e. $\lambda \in \mathbb{R}$ we conclude

$$
\operatorname{Im}(\log (N(z)))=\int_{\mathbb{R}} \frac{\operatorname{Im}(z)}{|\lambda-z|^{2}} \Xi(\lambda) d \lambda \in \mathfrak{S}_{1}(\mathcal{G})
$$


for all $z \in \mathbb{C} \backslash \mathbb{R}$. The last assertion on the existence of the $\operatorname{limit} \operatorname{Im}(\log (N(\lambda+i 0)))$ for a.e. $\lambda \in \mathbb{R}$ in $\mathfrak{S}_{1}(\mathcal{G})$ is an immediate consequence of (3.31) and well-known results in $[10,60,61]$ (cf. [26, Theorem 2.2(iii)]).

The following lemma will be useful in the proof of our main result, Theorem 4.1, in the next section; it also provides a sufficient condition for the assumption (3.23) in Proposition 3.4.

Lemma 3.5 Let $N: \mathbb{C} \backslash \mathbb{R} \rightarrow \mathcal{L}(\mathcal{G})$ be a Nevanlinna function such that $N(z)^{-1} \in$ $\mathcal{L}(\mathcal{G})$ for some, and hence for all $z \in \mathbb{C} \backslash \mathbb{R}$. Let $\ell \in \mathbb{N}$ and assume that

$$
\frac{d^{j}}{d z^{j}} N(z) \in \mathfrak{S}_{\frac{l}{j}}(\mathcal{G}), \quad j=1, \ldots, \ell,
$$

holds for all $z \in \mathbb{C} \backslash \mathbb{R}$. Then

$$
\frac{d^{\ell}}{d z^{\ell}} \log (N(z)) \in \mathfrak{S}_{1}(\mathcal{G}) \quad \text { and } \quad \frac{d^{\ell-1}}{d z^{\ell-1}}\left(N(z)^{-1} \frac{d}{d z} N(z)\right) \in \mathfrak{S}_{1}(\mathcal{G})
$$

and

$$
\operatorname{tr}_{\mathcal{G}}\left(\frac{d^{\ell-1}}{d z^{\ell-1}}\left(N(z)^{-1} \frac{d}{d z} N(z)\right)\right)=\operatorname{tr}_{\mathcal{G}}\left(\frac{d^{\ell}}{d z^{\ell}} \log (N(z))\right)
$$

hold for all $z \in \mathbb{C} \backslash \mathbb{R}$.

Furthermore, if $N$ admits an analytic continuation by reflection with respect to an open interval $I \subset \mathbb{R}$ such that $\sigma(N(z)) \subset(\varepsilon, \infty)$ for some $\varepsilon>0$ and all $z \in I$, and (3.32) is satisfied for $z \in I$, then also the assertions (3.33) and (3.34) are valid for all $z \in I$.

Proof We prove Lemma 3.5 for the case $\ell=1$ and leave the induction step to the reader. Assume that

$$
\frac{d}{d z} N(z) \in \mathfrak{S}_{1}(\mathcal{G})
$$

holds for $z \in \mathbb{C}_{+}$(the proof works also for $z \in I$ if $N$ admits an analytic continuation by reflection with respect to $I$ and $\sigma(N(z)) \subset(\varepsilon, \infty)$ holds for some $\varepsilon>0$ and all $z \in I$ ). One notes that $N(z)^{-1} \in \mathcal{L}(\mathcal{G})$ implies the second assertion in (3.33) for $\ell=1$. In addition, one observes that $\log (N(z))$ is well-defined and analytic for $z \in \mathbb{C}_{+}$according to (3.12) and Theorem 3.3. Since $0 \in \rho(N(z))$ and

$$
\left\|\left(N(z)+i \lambda I_{\mathcal{G}}\right)^{-1}\right\|_{\mathcal{L}(\mathcal{G})} \leq \lambda^{-1}, \quad \lambda>0
$$

(cf. the proof of Lemma 3.1 and [26, Proof of Lemma 2.6(i)]), it follows by the dominated convergence theorem that

$$
\begin{aligned}
& \frac{d}{d z}(\log (N(z)) \varphi, \psi)_{\mathcal{G}} \\
& \quad=i \int_{0}^{\infty}\left(\left(N(z)+i \lambda I_{\mathcal{G}}\right)^{-1}\left(\frac{d}{d z} N(z)\right)\left(N(z)+i \lambda I_{\mathcal{G}}\right)^{-1} \varphi, \psi\right)_{\mathcal{G}} d \lambda
\end{aligned}
$$


holds for all $\varphi, \psi \in \mathcal{G}$ and all $z \in \mathbb{C}_{+}$, and hence

$$
\begin{aligned}
& \frac{d}{d z} \log (N(z)) \\
& \quad=i \int_{0}^{\infty}\left(N(z)+i \lambda I_{\mathcal{G}}\right)^{-1}\left(\frac{d}{d z} N(z)\right)\left(N(z)+i \lambda I_{\mathcal{G}}\right)^{-1} d \lambda, \quad z \in \mathbb{C}_{+} .
\end{aligned}
$$

The assumption (3.35) yields

$$
\left(N(z)+i \lambda I_{\mathcal{G}}\right)^{-1}\left(\frac{d}{d z} N(z)\right)\left(N(z)+i \lambda I_{\mathcal{G}}\right)^{-1} \in \mathfrak{S}_{1}(\mathcal{G}), \quad \lambda \geq 0 .
$$

From (3.36) and the properties of the trace class norm $\|\cdot\|_{\mathfrak{S}_{1}(\mathcal{G})}$ one gets

$$
\left\|\left(N(z)+i \lambda I_{\mathcal{G}}\right)^{-1}\left(\frac{d}{d z} N(z)\right)\left(N(z)+i \lambda I_{\mathcal{G}}\right)^{-1}\right\|_{\mathfrak{S}_{1}(\mathcal{G})} \leq \frac{1}{\lambda^{2}}\left\|\frac{d}{d z} N(z)\right\|_{\mathfrak{S}_{1}(\mathcal{G})},
$$

$\lambda>0$, and hence the integral in (3.37) exists in trace class norm, that is, the first assertion in (3.33) holds for $\ell=1$. In order to prove (3.34) for $\ell=1$ we use (3.37) and cyclicity of the trace (i.e., $\operatorname{tr}_{\mathcal{G}}(C D)=\operatorname{tr}_{\mathcal{G}}(D C)$ whenever $C, D \in \mathcal{L}(\mathcal{G})$ such that $\left.C D, D C \in \mathfrak{S}_{1}(\mathcal{G})\right)$ and obtain

$$
\begin{aligned}
\operatorname{tr}_{\mathcal{G}}\left(\frac{d}{d z} \log (N(z))\right) & =\operatorname{tr}_{\mathcal{G}}\left(i \int_{0}^{\infty}\left(N(z)+i \lambda I_{\mathcal{G}}\right)^{-1}\left(\frac{d}{d z} N(z)\right)\left(N(z)+i \lambda I_{\mathcal{G}}\right)^{-1} d \lambda\right) \\
& =i \int_{0}^{\infty} \operatorname{tr}_{\mathcal{G}}\left(\left(N(z)+i \lambda I_{\mathcal{G}}\right)^{-2} \frac{d}{d z} N(z)\right) d \lambda \\
& =\int_{0}^{\infty} \operatorname{tr}_{\mathcal{G}}\left(-\frac{d}{d \lambda}\left(N(z)+i \lambda I_{\mathcal{G}}\right)^{-1} \frac{d}{d z} N(z)\right) d \lambda \\
& =-\int_{0}^{\infty} \frac{d}{d \lambda} \operatorname{tr}_{\mathcal{G}}\left(\left(N(z)+i \lambda I_{\mathcal{G}}\right)^{-1} \frac{d}{d z} N(z)\right) d \lambda \\
& =\operatorname{tr}_{\mathcal{G}}\left(N(z)^{-1} \frac{d}{d z} N(z)\right) .
\end{aligned}
$$

Here we have used $\lim _{\lambda \rightarrow+\infty} \operatorname{tr}_{\mathcal{G}}\left(\left(N(z)+i \lambda I_{\mathcal{G}}\right)^{-1} \frac{d}{d z} N(z)\right)=0$ in the last step, which follows from

$$
\left\|\left(N(z)+i \lambda I_{\mathcal{G}}\right)^{-1} \frac{d}{d z} N(z)\right\|_{\mathfrak{S}_{1}(\mathcal{G})} \leq \frac{1}{\lambda}\left\|\frac{d}{d z} N(z)\right\|_{\mathfrak{S}_{1}(\mathcal{G})}, \quad \lambda>0 .
$$




\section{A representation of the spectral shift function in terms of the Weyl function}

Let $A$ and $B$ be self-adjoint operators in a separable Hilbert space $\mathfrak{H}$ and assume that the closed symmetric operator $S=A \cap B$, that is,

$$
S f=A f=B f, \quad \operatorname{dom}(S)=\{f \in \operatorname{dom}(A) \cap \operatorname{dom}(B) \mid A f=B f\},
$$

is densely defined. According to Proposition 2.4 we can choose a quasi boundary triple $\left\{\mathcal{G}, \Gamma_{0}, \Gamma_{1}\right\}$ with $\gamma$-field $\gamma$ and Weyl function $M$ such that

$$
A=T \uparrow \operatorname{ker}\left(\Gamma_{0}\right) \quad \text { and } \quad B=T \uparrow \operatorname{ker}\left(\Gamma_{1}\right) \text {, }
$$

and

$$
\left(B-z I_{\mathfrak{H}}\right)^{-1}-\left(A-z I_{\mathfrak{H}}\right)^{-1}=-\gamma(z) M(z)^{-1} \gamma(\bar{z})^{*}, \quad z \in \rho(A) \cap \rho(B) .
$$

In the next theorem we find an explicit expression for a spectral shift function of the pair $\{A, B\}$ in terms of the Weyl function $M$, see [49, Theorem 1] for the case that the difference of (the first powers of) the resolvents $A$ and $B$ is a rank one operator, [7, Theorem 4.1] for the finite-rank case, and [56, Theorem 3.4 and Remark 3.5] for a different representation via a perturbation determinant involving the Weyl function and boundary parameters of an ordinary boundary triple. In the present situation of infinite dimensional perturbations and differences of higher powers of resolvents a much more careful analysis is necessary, in particular, the properties of the logarithm of operator-valued Nevanlinna functions discussed in Sect. 3 will play an essential role. In Theorem 4.1 an implicit sign condition on the perturbation is imposed via the resolvents which leads to a nonnegative spectral shift function; this condition will be weakend afterwards (cf. (4.25) and (4.29)). In the special case that $A$ and $B$ are semibounded operators the sign condition (4.4) is equivalent to the inequality $\mathfrak{t}_{A} \leq \mathfrak{t}_{B}$ of the semibounded closed quadratic forms $\mathfrak{t}_{A}$ and $\mathfrak{t}_{B}$ corresponding to $A$ and $B$. In order to ensure that for some $k \in \mathbb{N}_{0}$ the difference of the $2 k+1$ th-powers of the resolvents of $A$ and $B$ is a trace class operator a set of $\mathfrak{S}_{p}$-conditions on the $\gamma$-field and the Weyl function are imposed. In the applications in Sects. 5 and 6 these conditions are satisfied.

Theorem 4.1 Let $A$ and $B$ be self-adjoint operators in a separable Hilbert space $\mathfrak{H}$ and assume that for some $\zeta_{0} \in \rho(A) \cap \rho(B) \cap \mathbb{R}$ the sign condition

$$
\left(A-\zeta_{0} I_{\mathfrak{H}}\right)^{-1} \geq\left(B-\zeta_{0} I_{\mathfrak{H}}\right)^{-1}
$$

holds. Let the closed symmetric operator $S=A \cap B$ in (4.1) be densely defined and let $\left\{\mathcal{G}, \Gamma_{0}, \Gamma_{1}\right\}$ be a quasi boundary triple with $\gamma$-field $\gamma$ and Weyl function $M$ such that (4.2), and hence also (4.3), hold. Assume that $M\left(z_{1}\right), M\left(z_{2}\right)^{-1}$ are bounded (not necessarily everywhere defined) operators in $\mathcal{G}$ for some $z_{1}, z_{2} \in \rho(A) \cap \rho(B)$ and 
that for some $k \in \mathbb{N}_{0}$, all $p, q \in \mathbb{N}_{0}$, and all $z \in \rho(A) \cap \rho(B)$,

$$
\begin{aligned}
& \left(\frac{d^{p}}{d z^{p}} \overline{\gamma(z)}\right) \frac{d^{q}}{d z^{q}}\left(M(z)^{-1} \gamma(\bar{z})^{*}\right) \in \mathfrak{S}_{1}(\mathfrak{H}), \quad p+q=2 k, \\
& \left(\frac{d^{q}}{d z^{q}}\left(M(z)^{-1} \gamma(\bar{z})^{*}\right)\right) \frac{d^{p}}{d z^{p}} \overline{\gamma(z)} \in \mathfrak{S}_{1}(\mathcal{G}), \quad p+q=2 k,
\end{aligned}
$$

and

$$
\frac{d^{j}}{d z^{j}} \overline{M(z)} \in \mathfrak{S}_{(2 k+1) / j}(\mathcal{G}), \quad j=1, \ldots, 2 k+1 .
$$

Then the following assertions (i) and (ii) hold:

(i) The difference of the $2 k+1$ th-powers of the resolvents of $A$ and $B$ is a trace class operator, that is,

$$
\left[\left(B-z I_{\mathfrak{H}}\right)^{-(2 k+1)}-\left(A-z I_{\mathfrak{H}}\right)^{-(2 k+1)}\right] \in \mathfrak{S}_{1}(\mathfrak{H})
$$

holds for all $z \in \rho(A) \cap \rho(B)$.

(ii) For any orthonormal basis $\left(\varphi_{j}\right)_{j \in J}$ in $\mathcal{G}$ the function

$$
\xi(\lambda)=\sum_{j \in J} \lim _{\varepsilon \downarrow 0} \pi^{-1}\left(\operatorname{Im}(\log (\overline{M(\lambda+i \varepsilon)})) \varphi_{j}, \varphi_{j}\right)_{\mathcal{G}} \quad \text { for a.e. } \lambda \in \mathbb{R}
$$

is a spectral shift function for the pair $\{A, B\}$ such that $\xi(\lambda)=0$ in an open neighborhood of $\zeta_{0}$; the function $\xi$ does not depend on the choice of the orthonormal basis $\left(\varphi_{j}\right)_{j \in J}$. In particular, the trace formula

$$
\operatorname{tr}_{\mathfrak{H}}\left(\left(B-z I_{\mathfrak{H}}\right)^{-(2 k+1)}-\left(A-z I_{\mathfrak{H}}\right)^{-(2 k+1)}\right)=-(2 k+1) \int_{\mathbb{R}} \frac{\xi(\lambda) d \lambda}{(\lambda-z)^{2 k+2}}
$$

is valid for all $z \in \rho(A) \cap \rho(B)$.

Proof Step 1 In this step we show that the Nevanlinna function $z \mapsto \overline{M(z)}$ satisfies the assumptions of Theorem 3.3 and admits an analytic continuation by reflection with respect to an open interval $I_{\zeta_{0}} \subset \mathbb{R}$, such that $\sigma(\overline{M(z)}) \subset(\varepsilon, \infty)$ for some $\varepsilon>0$ and all $z \in I_{\zeta_{0}}$, where $I_{\zeta_{0}} \subset \rho(A) \cap \rho(B)$ is a suitable small open interval in $\mathbb{R}$ with $\zeta_{0} \in I_{\zeta_{0}}$. Hence by Theorem 3.3 there exists a weakly Lebesgue measurable operator function $\lambda \mapsto \Xi(\lambda) \in \mathcal{L}(\mathcal{G})$ on $\mathbb{R}$ such that

$$
\Xi(\lambda)=\Xi(\lambda)^{*} \text { and } \quad 0 \leq \Xi(\lambda) \leq I_{\mathcal{G}} \quad \text { for a.e. } \lambda \in \mathbb{R} \text {, }
$$

and the Nevanlinna function $\log (\bar{M})$ admits an integral representation of the form

$$
\log (\overline{M(z)})=\operatorname{Re}(\log (\overline{M(i)}))+\int_{\mathbb{R}}\left(\frac{1}{\lambda-z}-\frac{\lambda}{1+\lambda^{2}}\right) \Xi(\lambda) d \lambda,
$$

valid for all $z \in(\mathbb{C} \backslash \mathbb{R}) \cup I_{\zeta_{0}}$, and $\Xi(\lambda)=0$ for a.e. $\lambda \in I_{\zeta_{0}}$. 
First, it follows from (2.8) and the assumption that $M\left(z_{1}\right)$ is bounded for some $z_{1} \in \rho(A)$ that $M(z)$ is bounded for all $z \in \rho(A)$ and hence the closures are bounded operators defined on $\mathcal{G}$, that is,

$$
\overline{M(z)} \in \mathcal{L}(\mathcal{G}), \quad z \in \rho(A), \quad \text { and } \operatorname{Im}(\overline{M(z)}) \geq 0, \quad z \in \mathbb{C}_{+},
$$

by (2.11). Since $-M^{-1}$ is the Weyl function corresponding to the quasi boundary triple $\left\{\mathcal{G}, \Gamma_{1},-\Gamma_{0}\right\}$, where $B=T \uparrow \operatorname{ker}\left(\Gamma_{1}\right)$ is self-adjoint according to (4.2), it follows from the assumption that $M\left(z_{2}\right)^{-1}$ is bounded for some $z_{2} \in \rho(B)$ that $M(z)^{-1}$ is bounded for all $z \in \rho(A) \cap \rho(B)$, that is,

$$
\overline{M(z)^{-1}} \in \mathcal{L}(\mathcal{G}) \text { for all } z \in \rho(A) \cap \rho(B) .
$$

Therefore, taking into account (4.12) and (4.13), it follows that the logarithm $z \mapsto$ $\log (\overline{M(z)}) \in \mathcal{L}(\mathcal{G})$ is well-defined by

$$
\log (\overline{M(z)}):=-i \int_{0}^{\infty}\left[\left(\overline{M(z)}+i \lambda I_{\mathcal{G}}\right)^{-1}-(1+i \lambda)^{-1} I_{\mathcal{G}}\right] d \lambda, \quad z \in \mathbb{C}_{+},
$$

and

$$
\log (\overline{M(z)}):=(\log (\overline{M(\bar{z})}))^{*}, \quad z \in \mathbb{C}_{-} ;
$$

see (3.12)-(3.13) in Sect. 3 and [26, Lemma 2.6]. We claim that the function $\bar{M}$ has the property

$$
\sigma(\overline{M(z)}) \subset(\varepsilon, \infty)
$$

for some $\varepsilon>0$ and all $z \in I_{\zeta_{0}}$, where $I_{\zeta_{0}}$ is a suitable small open interval in $\mathbb{R}$ with $\zeta_{0} \in I_{\zeta_{0}}$. In fact, due to (4.3) and the sign condition (4.4), one has

$$
\begin{aligned}
0 & \leq\left(\left(A-\zeta_{0} I_{\mathfrak{H}}\right)^{-1} f-\left(B-\zeta_{0} I_{\mathfrak{H}}\right)^{-1} f, f\right)_{\mathfrak{H}} \\
& =\left(M\left(\zeta_{0}\right)^{-1} \gamma\left(\zeta_{0}\right)^{*} f, \gamma\left(\zeta_{0}\right)^{*} f\right)_{\mathcal{G}}, \quad f \in \mathfrak{H},
\end{aligned}
$$

and since $\operatorname{ran}\left(\gamma\left(\zeta_{0}\right)^{*}\right)$ is dense in $\mathcal{G}$ (see (2.3)), it follows that the bounded operator $M\left(\zeta_{0}\right)^{-1}$ is nonnegative. The same is true for $M\left(\zeta_{0}\right)$ and the closure $\overline{M\left(\zeta_{0}\right)}$, and from (4.13) one concludes $\sigma\left(\overline{M\left(\zeta_{0}\right)}\right) \subset(\varepsilon, \infty)$ for some $\varepsilon>0$. Since $\zeta_{0} \in \rho(A) \cap \rho(B)$ the Nevanlinna function $\bar{M}$ admits an analytic continuation by reflection with respect to a real neighborhood of $\zeta_{0}$, and it follows that (4.16) holds for all $\lambda$ in a sufficiently small interval $I_{\zeta_{0}} \subset \rho(A) \cap \rho(B) \cap \mathbb{R}$ with $\zeta_{0} \in I_{\zeta_{0}}$.

Step 2 In this step we show that for $z \in(\mathbb{C} \backslash \mathbb{R}) \cup I_{\zeta_{0}}$, the trace class property (4.8) holds, and that

$$
\operatorname{tr}_{\mathfrak{H}}\left(\left(B-z I_{\mathfrak{H}}\right)^{-(2 k+1)}-\left(A-z I_{\mathfrak{H}}\right)^{-(2 k+1)}\right)=\operatorname{tr}_{\mathcal{G}}\left(\frac{-1}{(2 k) !} \frac{d^{2 k+1}}{d z^{2 k+1}} \log (\overline{M(z)})\right)
$$


In fact, for $z \in(\mathbb{C} \backslash \mathbb{R}) \cup I_{\zeta_{0}}$ one computes

$$
\begin{aligned}
(B & \left.-z I_{\mathfrak{H}}\right)^{-(2 k+1)}-\left(A-z I_{\mathfrak{H}}\right)^{-(2 k+1)} \\
& =\frac{1}{(2 k) !} \frac{d^{2 k}}{d z^{2 k}}\left(\left(B-z I_{\mathfrak{H}}\right)^{-1}-\left(A-z I_{\mathfrak{H}}\right)^{-1}\right) \\
& =\frac{-1}{(2 k) !} \frac{d^{2 k}}{d z^{2 k}}\left(\gamma(z) M(z)^{-1} \gamma(\bar{z})^{*}\right)=\frac{-1}{(2 k) !} \frac{d^{2 k}}{d z^{2 k}}\left(\overline{\gamma(z)} M(z)^{-1} \gamma(\bar{z})^{*}\right) \\
& =\frac{-1}{(2 k) !} \sum_{\substack{p+q=2 k \\
p, q \geqslant 0}}\left(\begin{array}{c}
2 k \\
p
\end{array}\right)\left(\frac{d^{p}}{d z^{p}} \overline{\gamma(z)}\right) \frac{d^{q}}{d z^{q}}\left(M(z)^{-1} \gamma(\bar{z})^{*}\right),
\end{aligned}
$$

and by assumption (4.5) each summand is a trace class operator; in the last step the product rule for holomorphic operator functions was applied, see, e.g. [6, (2.6)]. This proves (4.8). Furthermore, making use of both assumptions (4.5) and (4.6), the cyclicity of the trace (see, e.g., [72, Theorem 7.11(b)]), and

$$
\frac{d}{d z} \overline{M(z)}=\gamma(\bar{z})^{*} \overline{\gamma(z)}, \quad z \in \rho(A)
$$

one obtains

$$
\begin{aligned}
& \operatorname{tr}_{\mathfrak{H}}\left(\left(B-z I_{\mathfrak{H}}\right)^{-(2 k+1)}-\left(A-z I_{\mathfrak{H}}\right)^{-(2 k+1)}\right) \\
& =\frac{-1}{(2 k) !} \sum_{\substack{p+q=2 k \\
p, q \geqslant 0}}\left(\begin{array}{c}
2 k \\
p
\end{array}\right) \operatorname{tr}_{\mathfrak{H}}\left(\left(\frac{d^{p}}{d z^{p}} \overline{\gamma(z)}\right) \frac{d^{q}}{d z^{q}}\left(M(z)^{-1} \gamma(\bar{z})^{*}\right)\right) \\
& =\frac{-1}{(2 k) !} \sum_{\substack{p+q=2 k \\
p, q \geqslant 0}}\left(\begin{array}{c}
2 k \\
p
\end{array}\right) \operatorname{tr}_{\mathcal{G}}\left(\left(\frac{d^{q}}{d z^{q}}\left(M(z)^{-1} \gamma(\bar{z})^{*}\right)\right) \frac{d^{p}}{d z^{p}} \overline{\gamma(z)}\right) \\
& =\operatorname{tr}_{\mathcal{G}}\left(\frac{-1}{(2 k) !} \frac{d^{2 k}}{d z^{2 k}}\left(M(z)^{-1} \gamma(\bar{z})^{*} \overline{\gamma(z)}\right)\right) \\
& =\operatorname{tr}_{\mathcal{G}}\left(\frac{-1}{(2 k) !} \frac{d^{2 k}}{d z^{2 k}}\left(\overline{M(z)^{-1}} \frac{d}{d z} \overline{M(z)}\right)\right) .
\end{aligned}
$$

Noting that assumption (4.7) and Lemma 3.5 with $\ell=2 k+1$ imply

$$
\frac{d^{2 k+1}}{d z^{2 k+1}} \log (\overline{M(z)}) \in \mathfrak{S}_{1}(\mathcal{G})
$$

and that

$$
\operatorname{tr}_{\mathcal{G}}\left(\frac{d^{2 k}}{d z^{2 k}}\left(\overline{M(z)^{-1}} \frac{d}{d z} \overline{M(z)}\right)\right)=\operatorname{tr}_{\mathcal{G}}\left(\frac{d^{2 k+1}}{d z^{2 k+1}} \log (\overline{M(z)})\right),
$$

one concludes the trace formula (4.17). 
Step 3 Now we complete the proof of Theorem 4.1. Since (4.19) is valid for all $z \in I_{\zeta_{0}}$ the assumption (3.23) in Proposition 3.4 is satisfied. It then follows from Proposition 3.4 that $0 \leq \Xi(\lambda) \in \mathfrak{S}_{1}(\mathcal{G})$ for a.e. $\lambda \in \mathbb{R}$ and

$$
\operatorname{tr}_{\mathcal{G}}(\Xi(\lambda))=\sum_{j \in J} \lim _{\varepsilon \downarrow 0} \pi^{-1}\left(\operatorname{Im}(\log (\overline{M(\lambda+i \varepsilon)})) \varphi_{j}, \varphi_{j}\right)_{\mathcal{G}}
$$

holds for any orthonormal basis $\left(\varphi_{j}\right)_{j \in J}$ in $\mathcal{G}$ and for a.e. $\lambda \in \mathbb{R}$. Furthermore, from (4.11) one obtains

$$
\frac{d^{2 k+1}}{d z^{2 k+1}} \log (\overline{M(z)})=(2 k+1) ! \int_{\mathbb{R}} \frac{1}{(\lambda-z)^{2 k+2}} \Xi(\lambda) d \lambda, \quad z \in(\mathbb{C} \backslash \mathbb{R}) \cup I_{\zeta_{0}},
$$

and hence

$$
\operatorname{tr}_{\mathfrak{H}}\left(\left(B-z I_{\mathfrak{H}}\right)^{-(2 k+1)}-\left(A-z I_{\mathfrak{H}}\right)^{-(2 k+1)}\right)=-(2 k+1) \int_{\mathbb{R}} \frac{\operatorname{tr}_{\mathcal{G}}(\Xi(\lambda)) d \lambda}{(\lambda-z)^{2 k+2}}
$$

for all $z \in(\mathbb{C} \backslash \mathbb{R}) \cup I_{\zeta_{0}}$ by (4.17). It also follows from (4.22) that

$$
\int_{\mathbb{R}} \frac{\operatorname{tr}_{\mathcal{G}}(\Xi(\lambda)) d \lambda}{(1+|\lambda|)^{2 k+2}}<\infty
$$

holds and together with (4.21)-(4.23) we conclude that the function

$$
\xi(\lambda):=\operatorname{tr}_{\mathcal{G}}(\Xi(\lambda))=\sum_{j \in J} \lim _{\varepsilon \downarrow 0} \pi^{-1}\left(\operatorname{Im}(\log (\overline{M(\lambda+i \varepsilon)})) \varphi_{j}, \varphi_{j}\right)_{\mathcal{G}} \quad \text { for a.e. } \lambda \in \mathbb{R}
$$

in (4.9) is a spectral shift function for the pair $\{A, B\}$. Next, since

$$
\operatorname{tr}_{\mathcal{G}}(\Xi(\lambda))=\sum_{j \in J}\left(\Xi(\lambda) \varphi_{j}, \varphi_{j}\right)_{\mathcal{G}}
$$

does not depend on the choice of the orthonormal basis $\left(\varphi_{j}\right)_{j \in J}$, it follows that the function $\xi$ does not depend on the choice of the orthonormal basis (cf. Proposition 3.4). Finally, since $\Xi(\lambda)=0$ for a.e. $\lambda \in I_{\zeta_{0}}$ by Theorem 3.3 it follows that $\xi(\lambda)=0$ for a.e. $\lambda \in I_{\zeta_{0}}$.

In the special case $k=0$ Theorem 4.1 can be slightly improved. Here the essential feature is that Proposition 3.4 can be applied under the assumption (3.25), so that the $\operatorname{limit} \operatorname{Im}(\log (\overline{M(\lambda+i 0)}))$ exists in $\mathfrak{S}_{1}(\mathcal{G})$ for a.e. $\lambda \in \mathbb{R}$.

Corollary 4.2 Let $A$ and $B$ be self-adjoint operators in a separable Hilbert space $\mathfrak{H}$ and assume that for some $\zeta_{0} \in \rho(A) \cap \rho(B) \cap \mathbb{R}$ the sign condition

$$
\left(A-\zeta_{0} I_{\mathfrak{H}}\right)^{-1} \geq\left(B-\zeta_{0} I_{\mathfrak{H}}\right)^{-1}
$$


holds. Assume that the closed symmetric operator $S=A \cap B$ in (4.1) is densely defined and let $\left\{\mathcal{G}, \Gamma_{0}, \Gamma_{1}\right\}$ be a quasi boundary triple with $\gamma$-field $\gamma$ and Weyl function $M$ such that (4.2), and hence also (4.3), hold. Assume that $M\left(z_{1}\right), M\left(z_{2}\right)^{-1}$ are bounded (not necessarily everywhere defined) operators in $\mathcal{G}$ for some $z_{1}, z_{2} \in \rho(A)$ and that $\overline{\gamma\left(z_{0}\right)} \in \mathfrak{S}_{2}(\mathcal{G}, \mathfrak{H})$ for some $z_{0} \in \rho(A)$. Then the following assertions ( $\left.i\right)$-(iii) hold:

(i) The difference of the resolvents of $A$ and $B$ is a trace class operator, that is,

$$
\left[\left(B-z I_{\mathfrak{H}}\right)^{-1}-\left(A-z I_{\mathfrak{H}}\right)^{-1}\right] \in \mathfrak{S}_{1}(\mathfrak{H})
$$

holds for all $z \in \rho(A) \cap \rho(B)$.

(ii) $\operatorname{Im}(\log (\overline{M(z)})) \in \mathfrak{S}_{1}(\mathcal{G})$ for all $z \in \mathbb{C} \backslash \mathbb{R}$ and the limit

$$
\operatorname{Im}(\log (\overline{M(\lambda+i 0)})):=\lim _{\varepsilon \downarrow 0} \operatorname{Im}(\log (\overline{M(\lambda+i \varepsilon)}))
$$

exists for a.e. $\lambda \in \mathbb{R}$ in $\mathfrak{S}_{1}(\mathcal{G})$.

(iii) The function

$$
\xi(\lambda)=\pi^{-1} \operatorname{tr}_{\mathcal{G}}(\operatorname{Im}(\log (\overline{M(\lambda+i 0)}))) \text { for a.e. } \lambda \in \mathbb{R},
$$

is a spectral shift function for the pair $\{A, B\}$ such that $\xi(\lambda)=0$ in an open neighborhood of $\zeta_{0}$ and the trace formula

$$
\operatorname{tr}_{\mathfrak{H}}\left(\left(B-z I_{\mathfrak{H}}\right)^{-1}-\left(A-z I_{\mathfrak{H}}\right)^{-1}\right)=-\int_{\mathbb{R}} \frac{\xi(\lambda) d \lambda}{(\lambda-z)^{2}}
$$

is valid for all $z \in \rho(A) \cap \rho(B)$.

Proof The assumption $\overline{\gamma\left(z_{0}\right)} \in \mathfrak{S}_{2}(\mathcal{G}, \mathfrak{H})$ for some $z_{0} \in \rho(A)$ implies $\overline{\gamma(z)} \in$ $\mathfrak{S}_{2}(\mathcal{G}, \mathfrak{H})$ for all $z \in \rho(A)$ by $(2.5)$ and hence also $\gamma(z)^{*} \in \mathfrak{S}_{2}(\mathfrak{H}, \mathcal{G})$ for all $z \in \rho(A)$. Since $M(z)^{-1}$ is bounded for all $z \in \rho(A) \cap \rho(B)$ (see (2.8)), conditions (4.5)-(4.6) in Theorem 4.1 are satisfied for $k=0$ and all $z \in \rho(A) \cap \rho(B)$. Furthermore,

$$
\frac{d}{d z} \overline{M(z)}=\gamma(z)^{*} \overline{\gamma(z)} \in \mathfrak{S}_{1}(\mathcal{G})
$$

by (2.12) and hence (4.7) holds for $k=0$. In particular, by Lemma 3.5 we have

$$
\frac{d}{d z} \log (\overline{M(z)}) \in \mathfrak{S}_{1}(\mathcal{G})
$$

In Step 3 of the proof of Theorem 4.1 we can now apply Proposition 3.4 under the assumption (3.25), so that (3.26) holds with $N(\lambda+i 0)$ replaced by $\overline{M(\lambda+i 0)}$. Now the assertions (i)-(iii) in Corollary 4.2 follow from Theorem 4.1 and Proposition 3.4. 
In the next step we replace the sign condition (4.4) in the assumptions in Theorem 4.1 by some weaker comparability condition. Again, let $A$ and $B$ be self-adjoint operators in a separable Hilbert space $\mathfrak{H}$ and assume that there exists a self-adjoint operator $C$ in $\mathfrak{H}$ such that

$$
\left(C-\zeta_{A} I_{\mathfrak{H}}\right)^{-1} \geq\left(A-\zeta_{A} I_{\mathfrak{H}}\right)^{-1} \quad \text { and } \quad\left(C-\zeta_{B} I_{\mathfrak{H}}\right)^{-1} \geq\left(B-\zeta_{B} I_{\mathfrak{H}}\right)^{-1}
$$

for some $\zeta_{A} \in \rho(A) \cap \rho(C) \cap \mathbb{R}$ and some $\zeta_{B} \in \rho(B) \cap \rho(C) \cap \mathbb{R}$, respectively. Assume that the closed symmetric operators $S_{A}=A \cap C$ and $S_{B}=B \cap C$ are both densely defined and choose quasi boundary triples $\left\{\mathcal{G}_{A}, \Gamma_{0}^{A}, \Gamma_{1}^{A}\right\}$ and $\left\{\mathcal{G}_{B}, \Gamma_{0}^{B}, \Gamma_{1}^{B}\right\}$ with $\gamma$-fields $\gamma_{A}, \gamma_{B}$ and Weyl functions $M_{A}, M_{B}$ for

$$
T_{A}=S_{A}^{*} \uparrow(\operatorname{dom}(A)+\operatorname{dom}(C)) \quad \text { and } \quad T_{B}=S_{B}^{*} \uparrow(\operatorname{dom}(B)+\operatorname{dom}(C))
$$

such that

$$
C=T_{A}\left\lceil\operatorname{ker}\left(\Gamma_{0}^{A}\right)=T_{B}\left\lceil\operatorname{ker}\left(\Gamma_{0}^{B}\right)\right.\right.
$$

and

$$
A=T_{A} \uparrow \operatorname{ker}\left(\Gamma_{1}^{A}\right) \text { and } B=T_{B} \uparrow \operatorname{ker}\left(\Gamma_{1}^{B}\right),
$$

(cf. Proposition 2.4). Next, assume that for some $k \in \mathbb{N}_{0}$, the conditions in Theorem 4.1 are satisfied for the $\gamma$-fields $\gamma_{A}, \gamma_{B}$ and the Weyl functions $M_{A}, M_{B}$. Then the difference of the $2 k+1$-th powers of the resolvents of $A$ and $C$, and $B$ and $C$ are trace class operators, and for orthonormal bases $\left(\varphi_{j}\right)_{j \in J}$ in $\mathcal{G}_{A}$ and $\left(\psi_{\ell}\right)_{\ell \in L}$ in $\mathcal{G}_{B}$ $(J, L \subseteq \mathbb{N}$ appropriate index sets),

$$
\begin{array}{ll}
\xi_{A}(\lambda)=\sum_{j \in J} \lim _{\varepsilon \downarrow 0} \pi^{-1}\left(\operatorname{Im}\left(\log \left(\overline{M_{A}(\lambda+i \varepsilon)}\right)\right) \varphi_{j}, \varphi_{j}\right)_{\mathcal{G}_{A}} \quad \text { for a.e. } \lambda \in \mathbb{R}, \\
\xi_{B}(\lambda)=\sum_{\ell \in L} \lim _{\varepsilon \downarrow 0} \pi^{-1}\left(\operatorname{Im}\left(\log \left(\overline{M_{B}(\lambda+i \varepsilon)}\right)\right) \psi_{\ell}, \psi_{\ell}\right)_{\mathcal{G}_{B}} \quad \text { for a.e. } \lambda \in \mathbb{R},
\end{array}
$$

are spectral shift functions for the pairs $\{C, A\}$ and $\{C, B\}$, respectively. It follows for $z \in \rho(A) \cap \rho(B) \cap \rho(C)$ that

$$
\operatorname{tr}_{\mathfrak{H}}\left(\left(B-z I_{\mathfrak{H}}\right)^{-(2 k+1)}-\left(A-z I_{\mathfrak{H}}\right)^{-(2 k+1)}\right)=-(2 k+1) \int_{\mathbb{R}} \frac{\left[\xi_{B}(\lambda)-\xi_{A}(\lambda)\right] d \lambda}{(\lambda-z)^{2 k+2}}
$$

and $\int_{\mathbb{R}} \frac{\left|\xi_{B}(\lambda)-\xi_{A}(\lambda)\right| d \lambda}{(1+|\lambda|)^{2 m+2}}<\infty$. Therefore,

$$
\xi(\lambda)=\xi_{B}(\lambda)-\xi_{A}(\lambda) \text { for a.e. } \lambda \in \mathbb{R},
$$

is a spectral shift function for the pair $\{A, B\}$, and in the special case where $\mathcal{G}_{A}=$ $\mathcal{G}_{B}:=\mathcal{G}$ and $\left(\varphi_{j}\right)_{j \in J}$ is an orthonormal basis in $\mathcal{G}$, one infers that

$$
\xi(\lambda)=\sum_{j \in J} \lim _{\varepsilon \downarrow 0} \pi^{-1}\left(\left(\operatorname{Im}\left(\log \left(\overline{M_{B}(\lambda+i \varepsilon)}\right)-\log \left(\overline{M_{A}(\lambda+i \varepsilon)}\right)\right) \varphi_{j}, \varphi_{j}\right)_{\mathcal{G}}\right.
$$

for a.e. $\lambda \in \mathbb{R}$. 
We emphasize that in contrast to the spectral shift function in Theorem 4.1, the spectral shift function $\xi$ in (4.29) and (4.30) is not necessarily nonnegative.

\section{Elliptic differential operators with Robin boundary conditions}

In this section we consider a uniformly elliptic formally symmetric second-order differential expression $\mathcal{L}$ on a bounded or unbounded domain in $\mathbb{R}^{n}$ with compact boundary, and we determine a spectral shift function for a pair $\left\{A_{\beta_{0}}, A_{\beta_{1}}\right\}$ consisting of two self-adjoint Robin-realizations of $\mathcal{L}$. We shall assume throughout this section that the following hypothesis holds.

Hypothesis 5.1 Let $n \in \mathbb{N}, n \geq 2$, and $\Omega \subseteq \mathbb{R}^{n}$ be nonempty and open such that its boundary $\partial \Omega$ is nonempty, $C^{\infty}$-smooth, and compact. Consider the differential expression

$$
\mathcal{L}=-\sum_{j, k=1}^{n}\left(\frac{\partial}{\partial x_{j}}\left(a_{j k} \frac{\partial}{\partial x_{k}}\right)\right)+a
$$

on $\Omega$, where the real-valued coefficients $a_{j k} \in C^{\infty}(\bar{\Omega})$ satisfy $a_{j k}(x)=a_{k j}(x)$ for all $x \in \bar{\Omega}$ and $j, k=1, \ldots, n$, their first partial derivatives are bounded in $\bar{\Omega}$, and $a \in C^{\infty}(\bar{\Omega})$ is a real-valued, bounded, measurable function. Furthermore, it is assumed that $\mathcal{L}$ is uniformly elliptic on $\bar{\Omega}$, that is, for some $C>0$,

$$
\sum_{j, k=1}^{n} a_{j k}(x) \xi_{j} \xi_{k} \geq C \sum_{k=1}^{n} \xi_{k}^{2}
$$

holds for all $\xi=\left(\xi_{1}, \ldots, \xi_{n}\right)^{\top} \in \mathbb{R}^{n}$ and $x \in \bar{\Omega}$.

We briefly recall the definition and some mapping properties of the Dirichlet and (oblique) Neumann trace maps associated with the differential expression $\mathcal{L}$. For a function $f \in C^{\infty}(\bar{\Omega})$ we denote its trace by $\gamma_{D} f=\left.f\right|_{\partial \Omega}$ and we set

$$
\gamma_{\nu} f=\left.\sum_{j, k=1}^{n} a_{j k} \mathfrak{n}_{j} \frac{\partial f}{\partial x_{k}}\right|_{\partial \Omega}, \quad f \in C^{\infty}(\bar{\Omega})
$$

where $\mathfrak{n}(x)=\left(\mathfrak{n}_{1}(x), \ldots, \mathfrak{n}_{n}(x)\right)^{\top}$ is the unit normal vector at $x \in \partial \Omega$ pointing out of the domain $\Omega$. Let $C_{0}^{\infty}(\bar{\Omega}):=\left\{\left.h\right|_{\bar{\Omega}} \mid h \in C_{0}^{\infty}\left(\mathbb{R}^{n}\right)\right\}$ and recall that the mapping $C_{0}^{\infty}(\bar{\Omega}) \ni f \mapsto\left\{\gamma_{D} f, \gamma_{\nu} f\right\}$ can be extended to a continuous surjective mapping

$$
H^{2}(\Omega) \ni f \mapsto\left\{\gamma_{D} f, \gamma_{\nu} f\right\} \in H^{3 / 2}(\partial \Omega) \times H^{1 / 2}(\partial \Omega),
$$

and that Green's second identity

$$
(\mathcal{L} f, g)_{L^{2}(\Omega)}-(f, \mathcal{L} g)_{L^{2}(\Omega)}=\left(\gamma_{D} f, \gamma_{\nu} g\right)_{L^{2}(\partial \Omega)}-\left(\gamma_{\nu} f, \gamma_{D} g\right)_{L^{2}(\partial \Omega)}
$$


is valid for all $f, g \in H^{2}(\Omega)$; cf. [53]. We will also use the fact that

$$
\gamma_{D} f \in H^{k-1 / 2}(\partial \Omega) \text { for all } f \in H^{k}(\Omega), k \in \mathbb{N}
$$

The following lemma is a variant of [5, Lemma 4.7]; it will be useful for the $\mathfrak{S}_{p^{-}}$ estimates in this and the next section.

Lemma 5.2 Let $\Omega \subseteq \mathbb{R}^{n}$ be as in Hypothesis 5.1, let $X \in \mathcal{L}\left(L^{2}(\Omega), H^{t}(\partial \Omega)\right)$, and assume that $\operatorname{ran}(X) \subseteq H^{s}(\partial \Omega)$ for some $s>t \geq 0$. Then $X$ is compact and

$$
X \in \mathfrak{S}_{r}\left(L^{2}(\Omega), H^{t}(\partial \Omega)\right) \text { for all } r>(n-1) /(s-t) .
$$

Assume that $\beta_{0} \in C^{1}(\partial \Omega)$ and $\beta_{1} \in C^{1}(\partial \Omega)$ are real-valued functions. For $p=$ 0,1 we consider the elliptic differential operators in $L^{2}(\Omega)$,

$$
A_{\beta_{p}} f=\mathcal{L} f, \quad \operatorname{dom}\left(A_{\beta_{p}}\right)=\left\{f \in H^{2}(\Omega) \mid \beta_{p} \gamma_{D} f=\gamma_{\nu} f\right\}
$$

which correspond to the densely defined, closed, semibounded quadratic forms

$$
\mathfrak{a}_{\beta_{p}}[f, g]=\sum_{j, k=1}^{n}\left(a_{j k} \frac{\partial f}{\partial x_{k}}, \frac{\partial g}{\partial x_{j}}\right)_{L^{2}(\Omega)}+(a f, g)_{L^{2}(\Omega)}-\left(\beta_{p} \gamma_{D} f, \gamma_{D} g\right)_{L^{2}(\partial \Omega)}
$$

defined on $H^{1}(\Omega) \times H^{1}(\Omega)$. Both operators $A_{\beta_{0}}$ and $A_{\beta_{1}}$ are self-adjoint in $L^{2}(\Omega)$ and semibounded from below. For $\beta \in \mathbb{R}$ we shall also make use of the self-adjoint Robin realization

$$
A_{\beta} f=\mathcal{L} f, \quad \operatorname{dom}\left(A_{\beta}\right)=\left\{f \in H^{2}(\Omega) \mid \beta \gamma_{D} f=\gamma_{\nu} f\right\},
$$

which corresponds to the densely defined, closed, semibounded quadratic form

$$
\mathfrak{a}_{\beta}[f, g]=\sum_{j, k=1}^{n}\left(a_{j k} \frac{\partial f}{\partial x_{k}}, \frac{\partial g}{\partial x_{j}}\right)_{L^{2}(\Omega)}+(a f, g)_{L^{2}(\Omega)}-\left(\beta \gamma_{D} f, \gamma_{D} g\right)_{L^{2}(\partial \Omega)}
$$

on $H^{1}(\Omega) \times H^{1}(\Omega)$.

Next, we define the Neumann-to-Dirichlet map associated to $\mathcal{L}$ as a densely defined operator in $L^{2}(\partial \Omega)$. First one notes that for $\beta_{0}=0$ in (5.6) (or $\beta=0$ in (5.8)) one obtains

$$
A_{N}:=A_{0}=A_{\beta_{0}},
$$

where $A_{N}$ denotes the self-adjoint Neumann realization of $\mathcal{L}$ in $L^{2}(\Omega)$. One recalls that for $\varphi \in H^{1 / 2}(\partial \Omega)$ and $z \in \rho\left(A_{N}\right)$, the boundary value problem

$$
\mathcal{L} f_{z}=z f_{z}, \quad \gamma_{\nu} f_{z}=\varphi,
$$


admits a unique solution $f_{z} \in H^{2}(\Omega)$; this follows, for instance, from (5.3) and $z \in \rho\left(A_{N}\right)$. The corresponding solution operator is denoted by

$$
P_{v}(z): L^{2}(\partial \Omega) \rightarrow L^{2}(\Omega), \quad \varphi \mapsto f_{z},
$$

and it is clear that $\operatorname{dom}\left(P_{v}(z)\right)=H^{1 / 2}(\partial \Omega)$ and $\operatorname{ran}\left(P_{v}(z)\right) \subseteq H^{2}(\Omega)$. For $z \in \rho\left(A_{N}\right)$ the Neumann-to-Dirichlet map associated to $\mathcal{L}$ is defined as

$$
\mathcal{N}(z): L^{2}(\partial \Omega) \rightarrow L^{2}(\partial \Omega), \quad \varphi \mapsto \gamma_{D} P_{v}(z) \varphi
$$

it maps the (oblique) Neumann boundary values $\gamma_{v} f_{z}$ of solutions $f_{z} \in H^{2}(\Omega)$ of (5.11) onto the Dirichlet boundary values $\gamma_{D} f_{z}$. It follows from the properties of the trace maps that

$$
\operatorname{dom}(\mathcal{N}(z))=H^{1 / 2}(\partial \Omega) \text { and } \quad \operatorname{ran}(\mathcal{N}(z)) \subseteq H^{3 / 2}(\partial \Omega)
$$

In the next theorem a spectral shift function for the pair $\left\{A_{\beta_{0}}, A_{\beta_{1}}\right\}$ is expressed in terms of the limits of the Neumann-to-Dirichlet map $\mathcal{N}(z)$ and the functions $\beta_{0}$ and $\beta_{1}$ in the boundary conditions of the Robin realizations $A_{\beta_{0}}$ and $A_{\beta_{1}}$. We mention that the trace class condition for the difference of the $2 k+1$-th powers of the resolvents was shown for $k=0$ in [4,34] and for $k \in \mathbb{N}$ in [6].

Theorem 5.3 Assume Hypothesis 5.1, let $A_{\beta_{0}}$ and $A_{\beta_{1}}$ be the self-adjoint Robin realizations of $\mathcal{L}$ in $L^{2}(\Omega)$ in (5.6), let $\beta \in \mathbb{R}$ such that $\beta_{p}(x)<\beta$ for all $x \in \partial \Omega$ and $p=0,1$ and let $A_{\beta}$ be the self-adjoint Robin realizations of $\mathcal{L}$ in (5.8). Furthermore, let

$\mathcal{M}_{p}(z)=\left(\beta-\beta_{p}\right)^{-1}\left(\beta_{p} \overline{\mathcal{N}(z)}-I_{L^{2}(\partial \Omega)}\right)\left(\beta \overline{\mathcal{N}(z)}-I_{L^{2}(\partial \Omega)}\right)^{-1}, \quad z \in \mathbb{C} \backslash \mathbb{R}, \quad j=1,2$,

where $\overline{\mathcal{N}(z)}$ denotes the closure in $L^{2}(\partial \Omega)$ of the Neumann-to-Dirichlet map associated with $\mathcal{L}$ in (5.13). Then the following assertions $(i)$ and (ii) hold for $k \in \mathbb{N}_{0}$ such that $k \geq(n-3) / 4$ :

(i) The difference of the $2 k+1$ th-powers of the resolvents of $A_{\beta_{0}}$ and $A_{\beta_{1}}$ is a trace class operator, that is,

$$
\left[\left(A_{\beta_{1}}-z I_{L^{2}(\Omega)}\right)^{-(2 k+1)}-\left(A_{\beta_{0}}-z I_{L^{2}(\Omega)}\right)^{-(2 k+1)}\right] \in \mathfrak{S}_{1}\left(L^{2}(\Omega)\right)
$$

holds for all $z \in \rho\left(A_{\beta_{0}}\right) \cap \rho\left(A_{\beta_{1}}\right)$.

(ii) For any orthonormal basis $\left(\varphi_{j}\right)_{j \in J}$ in $L^{2}(\partial \Omega)$ the function

$$
\xi(\lambda)=\sum_{j \in J} \lim _{\varepsilon \downarrow 0} \pi^{-1}\left(\left(\operatorname{Im}\left(\log \left(\mathcal{M}_{1}(\lambda+i \varepsilon)\right)-\log \left(\mathcal{M}_{0}(\lambda+i \varepsilon)\right)\right)\right) \varphi_{j}, \varphi_{j}\right)_{L^{2}(\partial \Omega)}
$$

for a.e. $\lambda \in \mathbb{R}$, is a spectral shift function for the pair $\left\{A_{\beta_{0}}, A_{\beta_{1}}\right\}$ such that $\xi(\lambda)=0$ for $\lambda<\min \left(\sigma\left(A_{\beta}\right)\right)$ and the trace formula 


$$
\begin{aligned}
& \operatorname{tr}_{L^{2}(\Omega)}\left(\left(A_{\beta_{1}}-z I_{L^{2}(\Omega)}\right)^{-(2 k+1)}-\left(A_{\beta_{0}}-z I_{L^{2}(\Omega)}\right)^{-(2 k+1)}\right)=-(2 k+1) \int_{\mathbb{R}} \frac{\xi(\lambda) d \lambda}{(\lambda-z)^{2 k+2}} \\
& \quad \text { is valid for all } z \in \rho\left(A_{\beta_{0}}\right) \cap \rho\left(A_{\beta_{1}}\right) .
\end{aligned}
$$

Proof The proof of Theorem 5.3 consists of three steps. In the first step we construct a suitable quasi boundary triple such that the self-adjoint operators $A_{\beta}$ and $A_{\beta_{1}}$ correspond to the kernels of the boundary mappings $\Gamma_{0}$ and $\Gamma_{1}$, and in the second and third step we show that the pair $\left\{A_{\beta}, A_{\beta_{1}}\right\}$ and the $\gamma$-field and Weyl function satisfy the assumptions in Theorem 4.1. The same reasoning applies to the pair $\left\{A_{\beta}, A_{\beta_{0}}\right\}$, and hence Theorem 4.1 can be applied to both pairs $\left\{A_{\beta}, A_{\beta_{1}}\right\}$ and $\left\{A_{\beta}, A_{\beta_{0}}\right\}$, which together with the considerations at the end of Sect. 4 yield the assertions in Theorem 5.3.

Step 1 The basic techniques in this step have been used in a similar framework, for instance, in $[2,3,5,8]$. We consider the closed symmetric operator $S=A_{\beta} \cap A_{\beta_{1}}$, which is given by

$$
S f=\mathcal{L} f, \quad \operatorname{dom}(S)=\left\{f \in H^{2}(\Omega) \mid \gamma_{D} f=\gamma_{\nu} f=0\right\}
$$

where we have used that $\beta-\beta_{1}(x) \neq 0$ for all $x \in \partial \Omega$. In this step we check that the operator

$$
T f=\mathcal{L} f, \quad \operatorname{dom}(T)=H^{2}(\Omega),
$$

satisfies $\bar{T}=S^{*}$ and that $\left\{L^{2}(\partial \Omega), \Gamma_{0}, \Gamma_{1}\right\}$, where

$$
\Gamma_{0} f=\beta \gamma_{D} f-\gamma_{\nu} f, \quad \Gamma_{1} f=\left(\beta-\beta_{1}\right)^{-1}\left(\beta_{1} \gamma_{D} f-\gamma_{\nu} f\right), \quad f \in \operatorname{dom}(T),
$$

is a quasi boundary triple for $T \subset S^{*}$ such that

$$
A_{\beta}=T \uparrow \operatorname{ker}\left(\Gamma_{0}\right) \text { and } A_{\beta_{1}}=T \uparrow \operatorname{ker}\left(\Gamma_{1}\right)
$$

and for all $z \in \rho\left(A_{\beta}\right) \cap \rho\left(A_{N}\right)$, where $A_{N}$ is the self-adjoint Neumann realization in (5.10), the corresponding $\gamma$-field $\gamma$ and Weyl function $M$ in $L^{2}(\partial \Omega)$ are given by

$$
\gamma(z)=P_{\nu}(z)\left(\beta \mathcal{N}(z)-I_{L^{2}(\partial \Omega)}\right)^{-1}, \quad \operatorname{dom}(\gamma(z))=H^{1 / 2}(\partial \Omega)
$$

and

$$
\begin{aligned}
& M(z)=\left(\beta-\beta_{1}\right)^{-1}\left(\beta_{1} \mathcal{N}(z)-I_{L^{2}(\partial \Omega)}\right)\left(\beta \mathcal{N}(z)-I_{L^{2}(\partial \Omega)}\right)^{-1} \\
& \operatorname{dom}(M(z))=H^{1 / 2}(\partial \Omega)
\end{aligned}
$$


We will use Theorem 2.2 for this purpose. For $f, g \in \operatorname{dom}(T)=H^{2}(\Omega)$ one obtains with the help of Green's identity (5.4),

$$
\begin{aligned}
\left(\Gamma_{1} f, \Gamma_{0} g\right)_{L^{2}(\partial \Omega)}-\left(\Gamma_{0} f, \Gamma_{1} g\right)_{L^{2}(\partial \Omega)} \\
=\left(\left(\beta-\beta_{1}\right)^{-1}\left(\beta_{1} \gamma_{D} f-\gamma_{\nu} f\right), \beta \gamma_{D} g-\gamma_{\nu} g\right)_{L^{2}(\partial \Omega)} \\
\quad-\left(\beta \gamma_{D} f-\gamma_{\nu} f,\left(\beta-\beta_{1}\right)^{-1}\left(\beta_{1} \gamma_{D} g-\gamma_{\nu} g\right)\right)_{L^{2}(\partial \Omega)} \\
=\left(\gamma_{D} f, \gamma_{\nu} g\right)_{L^{2}(\partial \Omega)}-\left(\gamma_{\nu} f, \gamma_{D} g\right)_{L^{2}(\partial \Omega)}=(\mathcal{L} f, g)_{L^{2}(\Omega)}-(f, \mathcal{L} g)_{L^{2}(\Omega)} \\
=(T f, g)_{L^{2}(\Omega)}-(f, T g)_{L^{2}(\Omega)},
\end{aligned}
$$

and hence condition $(i)$ in Theorem 2.2 holds. Since

$$
\left(\begin{array}{l}
\Gamma_{0} f \\
\Gamma_{1} f
\end{array}\right)=\left(\begin{array}{cc}
\beta & -I_{L^{2}(\partial \Omega)} \\
\beta_{1}\left(\beta-\beta_{1}\right)^{-1} & -\left(\beta-\beta_{1}\right)^{-1}
\end{array}\right)\left(\begin{array}{l}
\gamma_{D} f \\
\gamma_{\nu} f
\end{array}\right), \quad f \in \operatorname{dom}(T),
$$

and the $2 \times 2$ operator matrix in (5.21) is an isomorphism in $L^{2}(\partial \Omega) \times L^{2}(\partial \Omega)$, it follows from (5.3) that $\operatorname{ran}\left(\Gamma_{0}, \Gamma_{1}\right)^{\top}$ is dense in $L^{2}(\partial \Omega) \times L^{2}(\partial \Omega)$. It is easy to see that $\operatorname{ker}\left(\Gamma_{0}\right) \cap \operatorname{ker}\left(\Gamma_{1}\right)$ is dense in $L^{2}(\Omega)$. Moreover, (5.18) is clear from the definition of $T$ and the boundary maps in (5.17). Hence also conditions ( $i$ i ) and (iii) in Theorem 2.2 are satisfied, and from (5.15)-(5.17) one obtains $S=T \uparrow\left(\operatorname{ker}\left(\Gamma_{0}\right) \cap \operatorname{ker}\left(\Gamma_{1}\right)\right)$. Thus Theorem 2.2 yields $\bar{T}=S^{*}$ and that $\left\{L^{2}(\partial \Omega), \Gamma_{0}, \Gamma_{1}\right\}$ is a quasi boundary triple for $S^{*}$ such that $(5.18)$ holds.

It remains to show the explicit form of the corresponding $\gamma$-field and Weyl function $M$ in (5.19) and (5.20), respectively. First of all it follows from (5.3) and the definition of $\Gamma_{0}$ in (5.17) that

$$
H^{1 / 2}(\partial \Omega)=\operatorname{ran}\left(\Gamma_{0}\right)=\operatorname{dom}(\gamma(z))=\operatorname{dom}(M(z)), \quad z \in \rho\left(A_{\beta}\right) .
$$

One notes that for $z \in \rho\left(A_{N}\right)$ and $f_{z} \in \operatorname{ker}\left(T-z I_{L^{2}(\Omega)}\right)$ one has $\mathcal{N}(z) \gamma_{\nu} f_{z}=\gamma_{D} f_{z}$ according to (5.13), and hence

$$
\left(\beta \mathcal{N}(z)-I_{L^{2}(\partial \Omega)}\right) \gamma_{\nu} f_{z}=\beta \gamma_{D} f_{z}-\gamma_{v} f_{z}=\Gamma_{0} f_{z}
$$

and

$$
\left(\beta-\beta_{1}\right)^{-1}\left(\beta_{1} \mathcal{N}(z)-I_{L^{2}(\partial \Omega)}\right) \gamma_{v} f_{z}=\left(\beta-\beta_{1}\right)^{-1}\left(\beta_{1} \gamma_{D} f_{z}-\gamma_{\nu} f_{z}\right)=\Gamma_{1} f_{z}
$$

by (5.17). The relation (5.22) also shows that $\operatorname{ker}\left(\beta \mathcal{N}(z)-I_{L^{2}(\partial \Omega)}\right)=\{0\}$ for $z \in$ $\rho\left(A_{\beta}\right) \cap \rho\left(A_{N}\right)$ and hence

$$
\gamma_{\nu} f_{z}=\left(\beta \mathcal{N}(z)-I_{L^{2}(\partial \Omega)}\right)^{-1} \Gamma_{0} f_{z}
$$


From this and (5.12) it follows that the $\gamma$-field corresponding to $\left\{L^{2}(\partial \Omega), \Gamma_{0}, \Gamma_{1}\right\}$ has the form (5.19). One also concludes from (5.24) and (5.23) that

$$
\left(\beta-\beta_{1}\right)^{-1}\left(\beta_{1} \mathcal{N}(z)-I_{L^{2}(\partial \Omega)}\right)\left(\beta \mathcal{N}(z)-I_{L^{2}(\partial \Omega)}\right)^{-1} \Gamma_{0} f_{z}=\Gamma_{1} f_{z}
$$

holds for all $f_{z} \in \operatorname{ker}\left(T-z I_{L^{2}(\Omega)}\right)$ and $z \in \rho\left(A_{\beta}\right) \cap \rho\left(A_{N}\right)$. Thus the Weyl function corresponding to the quasi boundary triple $\left\{L^{2}(\partial \Omega), \Gamma_{0}, \Gamma_{1}\right\}$ has the form (5.20).

Step 2 In this step we verify that the pair $\left\{A_{\beta}, A_{\beta_{1}}\right\}$ satisfies the sign condition (4.4) and that the values of Weyl function and its inverse are bounded operators; see the assumptions of Theorem 4.1 .

The assumption $\beta>\beta_{1}(x)$ shows that the semibounded quadratic forms $\mathfrak{a}_{\beta}$ and $\mathfrak{a}_{\beta_{1}}$ in (5.7) and (5.9) corresponding to $A_{\beta}$ and $A_{\beta_{1}}$ satisfy the inequality $\mathfrak{a}_{\beta} \leq \mathfrak{a}_{\beta_{1}}$. Hence $\min \left(\sigma\left(A_{\beta}\right)\right) \leq \min \left(\sigma\left(A_{\beta_{1}}\right)\right)$ and for $\zeta<\min \left(\sigma\left(A_{\beta}\right)\right)$ the forms $\mathfrak{a}_{\beta}-\zeta$ and $\mathfrak{a}_{\beta_{1}}-\zeta$ are both nonnegative, satisfy the inequality $\mathfrak{a}_{\beta}-\zeta \leq \mathfrak{a}_{\beta_{1}}-\zeta$, and hence the resolvents of the corresponding nonnegative self-adjoint operators $A_{\beta}-\zeta I_{L^{2}(\Omega)}$ and $A_{\beta_{1}}-\zeta I_{L^{2}(\Omega)}$ satisfy the inequality

$$
\left(A_{\beta}-\zeta I_{L^{2}(\Omega)}\right)^{-1} \geq\left(A_{\beta_{1}}-\zeta I_{L^{2}(\Omega)}\right)^{-1}, \quad \zeta<\min \left(\sigma\left(A_{\beta}\right)\right)
$$

(see, e.g., [41, Chapter VI, § 2.6] or [15, Chapter 10, §2-Theorem 6]). Thus the sign condition (4.4) in the assumptions of Theorem 4.1 holds.

Next we prove that

$$
\begin{aligned}
M\left(z_{1}\right) & =\left(\beta-\beta_{1}\right)^{-1}\left(\beta_{1} \mathcal{N}\left(z_{1}\right)-I_{L^{2}(\partial \Omega)}\right)\left(\beta \mathcal{N}\left(z_{1}\right)-I_{L^{2}(\partial \Omega)}\right)^{-1}, \\
M\left(z_{2}\right)^{-1} & =\left(\beta \mathcal{N}\left(z_{2}\right)-I_{L^{2}(\partial \Omega)}\right)\left(\beta_{1} \mathcal{N}\left(z_{2}\right)-I_{L^{2}(\partial \Omega)}\right)^{-1}\left(\beta-\beta_{1}\right),
\end{aligned}
$$

are bounded operators for some $z_{1}, z_{2} \in \mathbb{C} \backslash \mathbb{R}$. According to [5, Lemma 4.4] the closure $\overline{\mathcal{N}(z)}, z \in \mathbb{C} \backslash \mathbb{R}$, of the Neumann-to-Dirichlet map in (5.13) in $L^{2}(\partial \Omega)$ is compact, and hence $\beta \mathcal{N}(z)-I_{L^{2}(\partial \Omega)}$ and $\beta_{1} \mathcal{N}(z)-I_{L^{2}(\partial \Omega)}$ are densely defined bounded operators in $L^{2}(\partial \Omega)$, and for $z \in \mathbb{C} \backslash \mathbb{R}$ their closures are

$$
\left[\beta \overline{\mathcal{N}(z)}-I_{L^{2}(\partial \Omega)}\right] \in \mathcal{L}\left(L^{2}(\partial \Omega)\right) \quad \text { and } \quad\left[\beta_{1} \overline{\mathcal{N}(z)}-I_{L^{2}(\partial \Omega)}\right] \in \mathcal{L}\left(L^{2}(\partial \Omega)\right)
$$

In order to see that

$$
Q(z):=\left(\beta \mathcal{N}(z)-I_{L^{2}(\partial \Omega)}\right)^{-1} \quad \text { and } \quad Q_{1}(z):=\left(\beta_{1} \mathcal{N}(z)-I_{L^{2}(\partial \Omega)}\right)^{-1}
$$

are bounded for $z \in \mathbb{C} \backslash \mathbb{R}$ we argue in a similar way as in the proof of [5, Lemma 4.4]: First, one notes that $\mathcal{N}(z) \subseteq \mathcal{N}(\bar{z})^{*}, z \in \mathbb{C} \backslash \mathbb{R}$, holds by (5.4), and this yields that also $Q(z) \subseteq Q(\bar{z})^{*}, z \in \mathbb{C} \backslash \mathbb{R}$. Hence the operator $Q(z)$ is closable in $L^{2}(\partial \Omega)$. Moreover, as $Q(z)$ is defined on $H^{1 / 2}(\partial \Omega)$ and maps into $H^{1 / 2}(\partial \Omega)$, it follows that $Q(z)$ is a closed operator in $H^{1 / 2}(\partial \Omega)$, and hence

$$
Q(z): H^{1 / 2}(\partial \Omega) \rightarrow H^{1 / 2}(\partial \Omega), \quad \varphi \mapsto Q(z) \varphi
$$


is bounded. Therefore, the dual operator

$$
Q(z)^{\prime}: H^{-1 / 2}(\partial \Omega) \rightarrow H^{-1 / 2}(\partial \Omega), \quad \psi \mapsto Q(z)^{\prime} \psi
$$

where $\left(Q(z)^{\prime} \psi\right)(\varphi)=\psi(Q(z) \varphi), \varphi \in H^{1 / 2}(\partial \Omega)$, is also bounded. One verifies that $Q(\bar{z})^{\prime}$ is an extension of $Q(z)$ and hence by interpolation and (5.27) and (5.28), the restriction

$$
Q(\bar{z})^{\prime} \Gamma_{L^{2}(\partial \Omega)}: L^{2}(\partial \Omega) \rightarrow L^{2}(\partial \Omega), \quad \phi \mapsto Q(\bar{z})^{\prime} \phi,
$$

of $Q(\bar{z})^{\prime}$ onto $L^{2}(\partial \Omega)$ is a bounded operator in $L^{2}(\partial \Omega)$ and an extension of $Q(z)$. Hence for all $z \in \mathbb{C} \backslash \mathbb{R}$ the operator $Q(z)$ is bounded in $L^{2}(\partial \Omega)$ and its closure is

$$
\overline{Q(z)}=\left(\beta \overline{\mathcal{N}(z)}-I_{L^{2}(\partial \Omega)}\right)^{-1} \in \mathcal{L}\left(L^{2}(\partial \Omega)\right), \quad z \in \mathbb{C} \backslash \mathbb{R} .
$$

The same reasoning with $Q(z)$ replaced by $Q_{1}(z)$ shows that for all $z \in \mathbb{C} \backslash \mathbb{R}$ the operator $Q_{1}(z)$ is bounded in $L^{2}(\partial \Omega)$ and

$$
\overline{Q_{1}(z)}=\left(\beta_{1} \overline{\mathcal{N}(z)}-I_{L^{2}(\partial \Omega)}\right)^{-1} \in \mathcal{L}\left(L^{2}(\partial \Omega)\right), \quad z \in \mathbb{C} \backslash \mathbb{R}
$$

Next, it follows that $M\left(z_{1}\right)$ and $M\left(z_{2}\right)^{-1}$ in (5.25) are bounded in $L^{2}(\partial \Omega)$ for $z_{1}, z_{2} \in$ $\mathbb{C} \backslash \mathbb{R}$ and the closure of $M(z)$ is given by

$$
\overline{M(z)}=\left(\beta-\beta_{1}\right)^{-1}\left(\beta_{1} \overline{\mathcal{N}(z)}-I_{L^{2}(\partial \Omega)}\right)\left(\beta \overline{\mathcal{N}(z)}-I_{L^{2}(\partial \Omega)}\right)^{-1}
$$

by (5.26) and (5.29). One notes that $\overline{M(z)}=\mathcal{M}_{1}(z)$ in the formulation of Theorem 5.3.

Step 3 In this step we verify that the $\gamma$-field and Weyl function corresponding to the quasi boundary triple $\left\{L^{2}(\partial \Omega), \Gamma_{0}, \Gamma_{1}\right\}$ in Step 1 satisfy the $\mathfrak{S}_{p}$-conditions in the assumptions of Theorem 4 .1 for dimensions $n \in \mathbb{N}, n \geq 2$, and $k \geq(n-3) / 4$, that is, we verify for all $p, q \in \mathbb{N}_{0}$ and all $z \in \rho\left(A_{\beta}\right) \cap \rho\left(A_{\beta_{1}}\right)$ the conditions

$$
\begin{aligned}
& \overline{\gamma(z)}^{(p)}\left(M(z)^{-1} \gamma(\bar{z})^{*}\right)^{(q)} \in \mathfrak{S}_{1}\left(L^{2}(\Omega)\right), \quad p+q=2 k, \\
& \left(M(z)^{-1} \gamma(\bar{z})^{*}\right)^{(q)} \overline{\gamma(z)}{ }^{(p)} \in \mathfrak{S}_{1}\left(L^{2}(\partial \Omega)\right), \quad p+q=2 k,
\end{aligned}
$$

and

$$
\frac{d^{j}}{d z^{j}} \overline{M(z)} \in \mathfrak{S}_{(2 k+1) / j}\left(L^{2}(\partial \Omega)\right), \quad j=1, \ldots, 2 k+1 .
$$

In the following we shall often use the smoothing property

$$
\left(A_{\beta}-z I_{L^{2}(\Omega)}\right)^{-1} f \in H^{k+2}(\Omega) \quad \text { for all } f \in H^{k}(\Omega), k \in \mathbb{N}_{0},
$$


of the resolvent of $A_{\beta}$, which follows, for instance, from [59, Theorem 4.18]. One notes that (2.2) and the definition of the boundary map $\Gamma_{1}$ in (5.17) yield

$$
\begin{aligned}
\gamma(\bar{z})^{*} f & =\Gamma_{1}\left(A_{\beta}-z I_{L^{2}(\Omega)}\right)^{-1} f \\
& =\left(\beta-\beta_{1}\right)^{-1}\left(\beta \gamma_{D}-\gamma_{\nu}+\left(\beta_{1}-\beta\right) \gamma_{D}\right)\left(A_{\beta}-z I_{L^{2}(\Omega)}\right)^{-1} f \\
& =\left(\beta-\beta_{1}\right)^{-1}\left(\beta \gamma_{D}-\gamma_{\nu}\right)\left(A_{\beta}-z I_{L^{2}(\Omega)}\right)^{-1} f-\gamma_{D}\left(A_{\beta}-z I_{L^{2}(\Omega)}\right)^{-1} f \\
& =-\gamma_{D}\left(A_{\beta}-z I_{L^{2}(\Omega)}\right)^{-1} f
\end{aligned}
$$

for all $z \in \rho\left(A_{\beta}\right)$ and $f \in L^{2}(\Omega)$. Here we have used in the last step that

$$
g=\left(A_{\beta}-z I_{L^{2}(\Omega)}\right)^{-1} f \in \operatorname{dom}\left(A_{\beta}\right)
$$

satisfies the boundary condition $\beta \gamma_{D} g-\gamma_{\nu} g=0$. It follows from (2.6) and (5.36) that

$$
\left(\gamma(\bar{z})^{*}\right)^{(q)}=q ! \gamma(\bar{z})^{*}\left(A_{\beta}-z I_{L^{2}(\Omega)}\right)^{-q}=-q ! \gamma_{D}\left(A_{\beta}-z I_{L^{2}(\Omega)}\right)^{-(q+1)},
$$

and hence, $\operatorname{ran}\left(\left(\gamma(\bar{z})^{*}\right)^{(q)}\right) \subset H^{2 q+3 / 2}(\partial \Omega)$ by (5.35) and (5.5). From Lemma 5.2 with $s=2 q+(3 / 2)$ and $t=0$ one concludes that

$$
\left(\gamma(\bar{z})^{*}\right)^{(q)} \in \mathfrak{S}_{r}\left(L^{2}(\Omega), L^{2}(\partial \Omega)\right), \quad r>(n-1) /[2 q+(3 / 2)],
$$

for all $z \in \rho\left(A_{\beta}\right), q \in \mathbb{N}_{0}$, and hence by (2.6) also

$$
\overline{\gamma(z)}^{(p)} \in \mathfrak{S}_{r}\left(L^{2}(\partial \Omega), L^{2}(\Omega)\right), \quad r>(n-1) /[2 p+(3 / 2)],
$$

for all $z \in \rho\left(A_{\beta}\right), p \in \mathbb{N}_{0}$. Furthermore,

$$
\frac{d^{j}}{d z^{j}} \overline{M(z)}=j ! \gamma(\bar{z})^{*}\left(A_{\beta}-z I_{L^{2}(\Omega)}\right)^{-(j-1)} \overline{\gamma(z)}, \quad j \in \mathbb{N},
$$

by (2.12) and with the help of (5.36) it follows in the same way as in (5.37) that

$$
\gamma(\bar{z})^{*}\left(A_{\beta}-z I_{L^{2}(\Omega)}\right)^{-(j-1)}=-\gamma_{D}\left(A_{\beta}-z I_{L^{2}(\Omega)}\right)^{-j} \in \mathfrak{S}_{x}\left(L^{2}(\Omega), L^{2}(\partial \Omega)\right)
$$

for $x>(n-1) /[2 j-(1 / 2)]$. Moreover, $\overline{\gamma(z)} \in \mathfrak{S}_{y}\left(L^{2}(\partial \Omega), L^{2}(\Omega)\right)$ for $y>$ $2(n-1) / 3$ by (5.38) and hence it follows from (5.39) and the well-known property $P Q \in \mathfrak{S}_{w}$ for $P \in \mathfrak{S}_{x}, Q \in \mathfrak{S}_{y}$, and $x^{-1}+y^{-1}=w^{-1}$, that

$$
\frac{d^{j}}{d z^{j}} \overline{M(z)} \in \mathfrak{S}_{w}\left(L^{2}(\partial \Omega), \quad w>(n-1) /(2 j+1), \quad z \in \rho\left(A_{\beta}\right), \quad j \in \mathbb{N} .\right.
$$


One observes that

$$
\frac{d}{d z} \overline{M(z)}^{-1}=-\overline{M(z)}^{-1}\left(\frac{d}{d z} \overline{M(z)}\right) \overline{M(z)}^{-1}, \quad z \in \rho\left(A_{\beta}\right) \cap \rho\left(A_{\beta_{1}}\right),
$$

that $\overline{M(z)}^{-1}$ is bounded, and by (5.40) that also

$$
\begin{aligned}
& \frac{d^{j}}{d z^{j}} \overline{M(z)}^{-1} \in \mathfrak{S}_{w}\left(L^{2}(\partial \Omega)\right), \quad w>(n-1) /(2 j+1), \\
& z \in \rho\left(A_{\beta}\right) \cap \rho\left(A_{\beta_{1}}\right), j \in \mathbb{N} ;
\end{aligned}
$$

we leave the formal induction step to the reader. Therefore,

$$
\begin{aligned}
\left(M(z)^{-1} \gamma(\bar{z})^{*}\right)^{(q)} & =\left(\overline{M(z)}^{-1} \gamma(\bar{z})^{*}\right)^{(q)}=\sum_{\substack{p+m=q \\
p, m \geqslant 0}}\left(\begin{array}{l}
q \\
p
\end{array}\right)\left(\overline{M(z)}^{-1}\right)^{(p)}\left(\gamma(\bar{z})^{*}\right)^{(m)} \\
& =\overline{M(z)}^{-1}\left(\gamma(\bar{z})^{*}\right)^{(q)}+\sum_{\substack{p+m=q \\
p>0, m \geq 0}}\left(\begin{array}{l}
q \\
p
\end{array}\right)\left(\overline{M(z)}^{-1}\right)^{(p)}\left(\gamma(\bar{z})^{*}\right)^{(m)}
\end{aligned}
$$

and one has $\overline{M(z)}^{-1}\left(\gamma(\bar{z})^{*}\right)^{(q)} \in \mathfrak{S}_{r}\left(L^{2}(\Omega), L^{2}(\partial \Omega)\right)$ for $r>(n-1) /[2 q+(3 / 2)]$ by (5.37) and each summand (and hence also the finite sum) on the right-hand side is in $\mathfrak{S}_{r}\left(L^{2}(\Omega), L^{2}(\partial \Omega)\right)$ for $r>(n-1) /[2 p+1+2 m+(3 / 2)]=(n-1) /[2 q+(5 / 2)]$, which follows from (5.41) and (5.38). Hence one has

$$
\left(M(z)^{-1} \gamma(\bar{z})^{*}\right)^{(q)} \in \mathfrak{S}_{r}\left(L^{2}(\Omega), L^{2}(\partial \Omega)\right)
$$

for $r>(n-1) /[2 q+(3 / 2)]$ and $z \in \rho\left(A_{\beta}\right) \cap \rho\left(A_{\beta_{1}}\right)$. From (5.38) and (5.42) one then concludes that

$$
\overline{\gamma(z)}^{(p)}\left(M(z)^{-1} \gamma(\bar{z})^{*}\right)^{(q)} \in \mathfrak{S}_{r}\left(L^{2}(\Omega)\right)
$$

for $r>(n-1) /[2(p+q)+3]=(n-1) /(4 k+3)$ and since $k \geq(n-3) / 4$, one has $1>(n-1) /(4 k+3)$, that is, the trace class condition $(5.32)$ is satisfied. The same argument shows that (5.33) is satisfied. Finally, (5.34) follows from (5.40) and the fact that $k \geq(n-3) / 4$ implies

$$
\frac{2 k+1}{j} \geq \frac{n-1}{2 j}>\frac{n-1}{2 j+1}, \quad j=1, \ldots, 2 k+1 .
$$

Hence the assumptions in Theorem 4.1 are satisfied with $S$ in (5.15), the quasi boundary triple in (5.17) and the corresponding $\gamma$-field and Weyl function in (5.19) and (5.20), respectively. Now Theorem 4.1 yields assertion $(i)$ in Theorem 5.3 with 
$A_{\beta_{0}}$ replaced by $A_{\beta}$ and for any orthonormal basis $\left(\varphi_{j}\right)_{j \in J}$ in $L^{2}(\partial \Omega)$ the function

$$
\xi_{1}(\lambda)=\sum_{j \in J} \lim _{\varepsilon \downarrow 0} \pi^{-1}\left(\operatorname{Im}\left(\log \left(\mathcal{M}_{1}(\lambda+i \varepsilon)\right)\right) \varphi_{j}, \varphi_{j}\right)_{L^{2}(\partial \Omega)} \quad \text { for a.e. } \lambda \in \mathbb{R},
$$

is a spectral shift function for the pair $\left\{A_{\beta}, A_{\beta_{1}}\right\}$ such that $\xi_{1}(\lambda)=0$ for $\lambda<$ $\min \left(\sigma\left(A_{\beta}\right)\right) \leq \min \left(\sigma\left(A_{\beta_{1}}\right)\right)$ and the trace formula

$$
\operatorname{tr}_{L^{2}(\Omega)}\left(\left(A_{\beta_{1}}-z I_{L^{2}(\Omega)}\right)^{-(2 k+1)}-\left(A_{\beta}-z I_{L^{2}(\Omega)}\right)^{-(2 k+1)}\right)=-(2 k+1) \int_{\mathbb{R}} \frac{\xi_{1}(\lambda) d \lambda}{(\lambda-z)^{2 k+2}}
$$

is valid for all $z \in \rho\left(A_{\beta}\right) \cap \rho\left(A_{\beta_{1}}\right)$.

The same construction as above with $\beta_{1}$ replaced by $\beta_{0}$ yields an analogous representation for a spectral shift function $\xi_{0}$ of the pair $\left\{A_{\beta}, A_{\beta_{0}}\right\}$. Finally it follows from the considerations in the end of Sect. 4 (see (4.29)) that

$$
\begin{aligned}
\xi(\lambda) & =\xi_{1}(\lambda)-\xi_{0}(\lambda) \\
& =\sum_{j \in J} \lim _{\varepsilon \downarrow 0} \pi^{-1}\left(\left(\operatorname{Im}\left(\log \left(\mathcal{M}_{1}(\lambda+i \varepsilon)\right)-\log \left(\mathcal{M}_{0}(\lambda+i \varepsilon)\right)\right)\right) \varphi_{j}, \varphi_{j}\right)_{L^{2}(\partial \Omega)}
\end{aligned}
$$

for a.e. $\lambda \in \mathbb{R}$ is a spectral shift function for the pair $\left\{A_{\beta_{0}}, A_{\beta_{1}}\right\}$ such that $\xi(\lambda)=0$ for $\lambda<\min \left(\sigma\left(A_{\beta}\right)\right) \leq \min \left(\sigma\left(A_{\beta_{p}}\right)\right), p=0,1$. This completes the proof of Theorem 5.3.

In space dimensions $n=2$ and $n=3$ one can choose $k=0$ in Theorem 5.3, and hence the resolvent difference of $A_{\beta_{1}}$ and $A_{\beta_{0}}$ is a trace class operator. In this situation Corollary 4.2 leads to the following slightly stronger statement.

Corollary 5.4 Let the assumptions be as in Theorem 5.3 and suppose that $n=2$ or $n=3$. Then the following assertions (i)-(iii) hold:

(i) The difference of the resolvents of $A_{\beta_{1}}$ and $A_{\beta_{0}}$ is a trace class operator, that is,

$$
\left[\left(A_{\beta_{1}}-z I_{L^{2}(\Omega)}\right)^{-1}-\left(A_{\beta_{0}}-z I_{L^{2}(\Omega)}\right)^{-1}\right] \in \mathfrak{S}_{1}\left(L^{2}(\Omega)\right)
$$

holds for all $z \in \rho\left(A_{\beta_{1}}\right) \cap \rho\left(A_{\beta_{0}}\right)$.

(ii) $\operatorname{Im}\left(\log \left(\mathcal{M}_{p}(z)\right)\right) \in \mathfrak{S}_{1}\left(L^{2}(\partial \Omega)\right)$ for all $z \in \mathbb{C} \backslash \mathbb{R}$ and $p=0$, 1 , and the limit

$$
\operatorname{Im}\left(\log \left(\mathcal{M}_{p}(\lambda+i 0)\right)\right):=\lim _{\varepsilon \downarrow 0} \operatorname{Im}\left(\log \left(\mathcal{M}_{p}(\lambda+i \varepsilon)\right)\right)
$$

exists for a.e. $\lambda \in \mathbb{R}$ and $p=0,1$ in $\mathfrak{S}_{1}\left(L^{2}(\partial \Omega)\right)$.

(iii) The function

$$
\xi(\lambda)=\pi^{-1} \operatorname{tr}_{L^{2}(\partial \Omega)}\left(\operatorname{Im}\left(\log \left(\mathcal{M}_{1}(\lambda+i 0)\right)-\log \left(\mathcal{M}_{0}(\lambda+i 0)\right)\right)\right) \quad \text { for a.e. } \lambda \in \mathbb{R}
$$


is a spectral shift function for the pair $\left\{A_{\beta_{0}}, A_{\beta_{1}}\right\}$ such that $\xi(\lambda)=0$ for $\lambda<$ $\min \left(\sigma\left(A_{\beta}\right)\right)$ and the trace formula

$$
\operatorname{tr}_{L^{2}(\Omega)}\left(\left(A_{\beta_{1}}-z I_{L^{2}(\Omega)}\right)^{-1}-\left(A_{\beta_{0}}-z I_{L^{2}(\Omega)}\right)^{-1}\right)=-(2 k+1) \int_{\mathbb{R}} \frac{\xi(\lambda) d \lambda}{(\lambda-z)^{2}}
$$

is valid for all $z \in \rho\left(A_{\beta_{0}}\right) \cap \rho\left(A_{\beta_{1}}\right)$.

\section{Schrödinger operators with compactly supported potentials}

In this section we determine a spectral shift function for the self-adjoint operators $\{-\Delta,-\Delta+V\}$ in $L^{2}\left(\mathbb{R}^{n}\right), n \in \mathbb{N}, n \geq 2$, where it is assumed that $V \in L^{\infty}\left(\mathbb{R}^{n}\right)$ is a compactly supported real-valued function. Thus we consider the self-adjoint operators

$$
A=-\Delta, \quad B=-\Delta+V, \quad \operatorname{dom}(A)=\operatorname{dom}(B)=H^{2}\left(\mathbb{R}^{n}\right),
$$

in $L^{2}\left(\mathbb{R}^{n}\right)$, and we fix an open ball $\mathcal{B}_{+} \subset \mathbb{R}^{n}$ such that $\operatorname{supp}(V) \subset \mathcal{B}_{+}$. The $n-1$ dimensional sphere $\partial \mathcal{B}_{+}$is denoted by $\mathcal{S}$. We shall also make use of the self-adjoint Dirichlet realizations

$$
A_{+}=-\Delta, \quad B_{+}=-\Delta+V, \quad \operatorname{dom}\left(A_{+}\right)=\operatorname{dom}\left(B_{+}\right)=H^{2}\left(\mathcal{B}_{+}\right) \cap H_{0}^{1}\left(\mathcal{B}_{+}\right),
$$

of $-\Delta$ and $-\Delta+V$ in $L^{2}\left(\mathcal{B}_{+}\right)$. Their spectra are discrete and bounded from below. The eigenvalue counting functions are denoted by $N\left(\cdot, A_{+}\right)$and $N\left(\cdot, B_{+}\right)$, respectively; recall that $N\left(\lambda, A_{+}\right)$and $N\left(\lambda, B_{+}\right)$stand for the total number of eigenvalues (multiplicities counted) of $A_{+}$and $B_{+}$in $(-\infty, \lambda), \lambda \in \mathbb{R}$.

The main ingredient in the proof of Theorem 6.1 below is a decoupling technique for the operators $A$ and $B$, where artificial Dirichlet boundary conditions on the sphere $\mathcal{S}$ will be imposed. We shall use the extension of the $L^{2}(\mathcal{S})$ scalar product onto the dual pair $H^{1 / 2}(\mathcal{S}) \times H^{-1 / 2}(\mathcal{S})$ via

$$
\langle\varphi, \psi\rangle=\left(l \varphi, \imath^{-1} \psi\right)_{L^{2}(\mathcal{S})}, \quad \varphi \in H^{1 / 2}(\mathcal{S}), \quad \psi \in H^{-1 / 2}(\mathcal{S})
$$

where $l$ is a uniformly positive self-adjoint operator in $L^{2}(\mathcal{S})$ defined on the dense subspace $H^{1 / 2}(\mathcal{S})$ (and in the following $\iota$ is regarded as an isomorphism from $H^{1 / 2}(\mathcal{S})$ onto $L^{2}(\mathcal{S})$ ), and $\iota^{-1}$ is the extension of $l^{-1}$ to an isomorphism from $H^{-1 / 2}(\mathcal{S})$ onto $L^{2}(\mathcal{S})$. A typical and convenient choice for $\imath$ is $\left(-\Delta_{\mathcal{S}}+I_{L^{2}(\mathcal{S})}\right)^{1 / 4}$, where $-\Delta_{\mathcal{S}}$ is the Laplace-Beltrami operator on the sphere $\mathcal{S}$; for other choices see also [8, Remark 5.3].

Since $\langle\cdot, \cdot\rangle$ in $(6.3)$ is an extension of the $L^{2}(\mathcal{S})$ scalar product, Green's identity can also be written in the form

$$
\left(-\Delta f_{+}, g_{+}\right)_{L^{2}\left(\mathcal{B}_{+}\right)}-\left(f_{+},-\Delta g_{+}\right)_{L^{2}\left(\mathcal{B}_{+}\right)}=\left\langle\gamma_{D}^{+} f_{+}, \gamma_{N}^{+} g_{+}\right\rangle-\left\langle\gamma_{N}^{+} f_{+}, \gamma_{D}^{+} g_{+}\right\rangle
$$


for $f_{+}, g_{+} \in H^{2}\left(\mathcal{B}_{+}\right)$. Here $\gamma_{D}^{+}$and $\gamma_{N}^{+}$denote the Dirichlet and Neumann trace operators in (5.3) (with $\Omega$ and $\partial \Omega$ replaced by $\mathcal{B}_{+}$and $\mathcal{S}$, respectively). Let $\mathcal{B}_{-}:=$ $\mathbb{R}^{n} \backslash \overline{\mathcal{B}}_{+}$and let $\gamma_{D}^{-}$and $\gamma_{N}^{-}$be the Dirichlet and Neumann trace operators on $\mathcal{B}_{-}$; the normal vector in the definition of $\gamma_{N}^{-}$is pointing in the outward direction of $\mathcal{B}_{-}$and hence opposite to the normal of $\mathcal{B}_{+}$. Besides (6.4) we also have the corresponding Green's identity on $\mathcal{B}_{-}$, that is,

$$
\left(-\Delta f_{-}, g_{-}\right)_{L^{2}\left(\mathcal{B}_{-}\right)}-\left(f_{-},-\Delta g_{-}\right)_{L^{2}\left(\mathcal{B}_{-}\right)}=\left\langle\gamma_{D}^{-} f_{-}, \gamma_{N}^{-} g_{-}\right\rangle-\left\langle\gamma_{N}^{-} f_{-}, \gamma_{D}^{-} g_{-}\right\rangle
$$

holds for all $f_{-}, g_{-} \in H^{2}\left(\mathcal{B}_{-}\right)$.

Next we define Dirichlet-to-Neumann maps associated to $-\Delta$ and $-\Delta+V$ on $\mathcal{B}_{+}$and $-\Delta$ on $\mathcal{B}_{-}$as operators from $H^{1 / 2}(\mathcal{S})$ to $H^{-1 / 2}(\mathcal{S})$. First, we recall that for $z \notin \sigma\left(A_{+}\right)$and $\varphi \in H^{1 / 2}(\mathcal{S})$ there exists a unique solution $f_{z} \in H^{1}\left(\mathcal{B}_{+}\right)$of the boundary value problem $-\Delta f_{z}=z f_{z}, \gamma_{D}^{+} f_{z}=\varphi$. The corresponding solution operator is $P_{+}(z): H^{1 / 2}(\mathcal{S}) \rightarrow H^{1}\left(\mathcal{B}_{+}\right), \varphi \mapsto f_{z}$, and for $z \notin \sigma\left(A_{+}\right)$, the Dirichletto-Neumann map $\mathcal{D}_{+}(z)$ associated to $-\Delta$ in $\mathcal{B}_{+}$is defined by

$$
\mathcal{D}_{+}(z): H^{1 / 2}(\mathcal{S}) \rightarrow H^{-1 / 2}(\mathcal{S}), \quad \varphi \mapsto \gamma_{N}^{+} P_{+}(z) \varphi
$$

Similarly, for $\zeta \notin \sigma\left(B_{+}\right)$and $\psi \in H^{1 / 2}(\mathcal{S})$, there exists a unique solution $g_{\zeta} \in$ $H^{1}\left(\mathcal{B}_{+}\right)$of the boundary value problem $(-\Delta+V) g_{\zeta}=\zeta g_{\zeta}, \gamma_{D}^{+} g_{\zeta}=\psi$. The corresponding solution operator is $P_{+}^{V}(\zeta): H^{1 / 2}(\mathcal{S}) \rightarrow H^{1}\left(\mathcal{B}_{+}\right), \psi \mapsto g_{\zeta}$, and for $\zeta \notin \sigma\left(B_{+}\right)$the Dirichlet-to-Neumann map $\mathcal{D}_{+}^{V}(\zeta)$ associated to $-\Delta+V$ in $\mathcal{B}_{+}$is defined by

$$
\mathcal{D}_{+}^{V}(\zeta): H^{1 / 2}(\mathcal{S}) \rightarrow H^{-1 / 2}(\mathcal{S}), \quad \psi \mapsto \gamma_{N}^{+} P_{+}^{V}(\zeta) \psi .
$$

Furthermore, for $\zeta^{\prime} \notin[0, \infty)$ and $\xi \in H^{1 / 2}(\mathcal{S})$ there exists a unique solution $h_{\zeta^{\prime}} \in$ $H^{1}\left(\mathcal{B}_{-}\right)$of the boundary value problem $-\Delta h_{\zeta^{\prime}}=\zeta^{\prime} h_{\zeta^{\prime}}, \gamma_{D}^{-} h_{\zeta^{\prime}}=\xi$. As above the solution operator is $P_{-}\left(\zeta^{\prime}\right): H^{1 / 2}(\mathcal{S}) \rightarrow H^{1}\left(\mathcal{B}_{-}\right), \xi \mapsto h_{\zeta^{\prime}}$, and for $\zeta^{\prime} \notin[0, \infty)$, the Dirichlet-to-Neumann map $\mathcal{D}_{-}\left(\zeta^{\prime}\right)$ associated to $-\Delta$ in $\mathcal{B}_{-}$is defined by

$$
\mathcal{D}_{-}\left(\zeta^{\prime}\right): H^{1 / 2}(\mathcal{S}) \rightarrow H^{-1 / 2}(\mathcal{S}), \quad \xi \mapsto \gamma_{N}^{-} P_{-}\left(\zeta^{\prime}\right) \xi
$$

One recalls that the Dirichlet-to-Neumann maps $\mathcal{D}_{+}(z), \mathcal{D}_{+}^{V}(\zeta)$, and $\mathcal{D}_{-}\left(\zeta^{\prime}\right)$ above are bounded operators from $H^{1 / 2}(\mathcal{S})$ to $H^{-1 / 2}(\mathcal{S})$. Moreover, for $z \in \mathbb{C} \backslash \mathbb{R}$, each of the Dirichlet-to-Neumann maps is boundedly invertible and the same is true for the sums

$$
\begin{aligned}
& \mathcal{D}_{+}(z)+\mathcal{D}_{-}(z): H^{1 / 2}(\mathcal{S}) \rightarrow H^{-1 / 2}(\mathcal{S}), \quad z \in \mathbb{C} \backslash \mathbb{R}, \\
& \mathcal{D}_{+}^{V}(z)+\mathcal{D}_{-}(z): H^{1 / 2}(\mathcal{S}) \rightarrow H^{-1 / 2}(\mathcal{S}), \quad z \in \mathbb{C} \backslash \mathbb{R} .
\end{aligned}
$$

Hence, the operators

$$
\begin{aligned}
\mathfrak{N}(z) & =\imath\left(\mathcal{D}_{+}(z)+\mathcal{D}_{-}(z)\right)^{-1} \tilde{\imath}: L^{2}(\mathcal{S}) \rightarrow L^{2}(\mathcal{S}), \quad z \in \mathbb{C} \backslash \mathbb{R}, \\
\mathfrak{N}_{V}(z) & =\imath\left(\mathcal{D}_{+}^{V}(z)+\mathcal{D}_{-}(z)\right)^{-1} \tilde{\imath}: L^{2}(\mathcal{S}) \rightarrow L^{2}(\mathcal{S}), \quad z \in \mathbb{C} \backslash \mathbb{R},
\end{aligned}
$$


are everywhere defined and bounded in $L^{2}(\mathcal{S})$.

In the next theorem we obtain a representation for a spectral shift function for $\{A, B\}$ in (6.1) via a decoupling technique and Theorem 4.1. The considerations in the beginning of Step 1 of the proof of Theorem 6.1 are similar as in [8, Section 5.2] and hence some details are omitted.

Theorem 6.1 Let $n \in \mathbb{N}, n \geq 2$, and $k \in \mathbb{N}, k>(n-2) / 4$, and suppose that $V \in L^{\infty}\left(\mathbb{R}^{n}\right)$ is real-valued with support in the open ball $\mathcal{B}_{+}$. In addition, let $\mathfrak{N}(z)$ and $\mathfrak{N}_{V}(z)$ be as in (6.6), and denote the eigenvalue counting functions of the Dirichlet operators $A_{+}$and $B_{+}$in $L^{2}\left(\mathcal{B}_{+}\right)$by $N\left(\cdot, A_{+}\right)$and $N\left(\cdot, B_{+}\right)$, respectively. Then the following assertions $(i)$ and (ii) hold:

(i) The difference of the $2 k+1$ th-powers of the resolvents of A and B is a trace class operator, that is,

$$
\left[\left(B-z I_{L^{2}\left(\mathbb{R}^{n}\right)}\right)^{-(2 k+1)}-\left(A-z I_{L^{2}\left(\mathbb{R}^{n}\right)}\right)^{-(2 k+1)}\right] \in \mathfrak{S}_{1}\left(L^{2}\left(\mathbb{R}^{n}\right)\right)
$$

holds for all $z \in \rho(B)=\rho(A) \cap \rho(B)$.

(ii) For any orthonormal basis $\left(\varphi_{j}\right)_{j \in J}$ in $L^{2}(\mathcal{S})$ the function

$$
\begin{aligned}
\xi(\lambda)= & \sum_{j \in J} \lim _{\varepsilon \downarrow 0} \pi^{-1}\left(\left(\operatorname{Im}\left(\log (\mathfrak{N}(\lambda+i \varepsilon))-\log \left(\mathfrak{N}_{V}(\lambda+i \varepsilon)\right)\right)\right) \varphi_{j}, \varphi_{j}\right)_{L^{2}(\mathcal{S})} \\
& +N\left(\lambda, B_{+}\right)-N\left(\lambda, A_{+}\right) \quad \text { for a.e. } \lambda \in \mathbb{R},
\end{aligned}
$$

is a spectral shift function for the pair $\{A, B\}$ such that $\xi(\lambda)=0$ for $\lambda<$ $\min (\sigma(B)) \leq 0$ and the trace formula

$$
\operatorname{tr}_{L^{2}\left(\mathbb{R}^{n}\right)}\left(\left(B-z I_{L^{2}\left(\mathbb{R}^{n}\right)}\right)^{-(2 k+1)}-\left(A-z I_{L^{2}\left(\mathbb{R}^{n}\right)}\right)^{-(2 k+1)}\right)=-(2 k+1) \int_{\mathbb{R}} \frac{\xi(\lambda) d \lambda}{(\lambda-z)^{2 k+2}}
$$

is valid for all $z \in \rho(B)=\rho(A) \cap \rho(B)$.

Proof Besides the self-adjoint operators $A=-\Delta$ and $B=-\Delta+V$ in (6.1), and the Dirichlet realizations $A_{+}=-\Delta$ and $B_{+}=-\Delta+V$ in $L^{2}\left(\mathcal{B}_{+}\right)$in (6.2) we shall also make use of the Dirichlet realization $A_{-}$of $-\Delta$ in $L^{2}\left(\mathcal{B}_{-}\right)$given by

$$
A_{-}=-\Delta, \quad \operatorname{dom}\left(A_{-}\right)=H^{2}\left(\mathcal{B}_{-}\right) \cap H_{0}^{1}\left(\mathcal{B}_{-}\right),
$$

as well as the orthogonal sums in $L^{2}\left(\mathbb{R}^{n}\right)=L^{2}\left(\mathcal{B}_{+}\right) \oplus L^{2}\left(\mathcal{B}_{-}\right)$,

$$
\begin{aligned}
& A_{D}:=\left(\begin{array}{cc}
A_{+} & 0 \\
0 & A_{-}
\end{array}\right) \quad \text { and } \quad B_{D}:=\left(\begin{array}{cc}
B_{+} & 0 \\
0 & A_{-}
\end{array}\right), \\
& \operatorname{dom}\left(A_{D}\right)=\operatorname{dom}\left(B_{D}\right)=\left(H^{2}\left(\mathcal{B}_{+}\right) \cap H_{0}^{1}\left(\mathcal{B}_{+}\right)\right) \times\left(H^{2}\left(\mathcal{B}_{-}\right) \cap H_{0}^{1}\left(\mathcal{B}_{-}\right)\right) .
\end{aligned}
$$


For any orthonormal basis $\left(\varphi_{j}\right)_{j \in J}$ in $L^{2}(\mathcal{S})$ we shall first prove the representation

$$
\xi_{A}(\lambda)=\sum_{j \in J} \lim _{\varepsilon \downarrow 0} \pi^{-1}\left(\operatorname{Im}(\log (\mathfrak{N}(\lambda+i \varepsilon))) \varphi_{j}, \varphi_{j}\right)_{L^{2}(\mathcal{S})}
$$

for a spectral shift function $\xi_{A}$ of the pair $\left\{A, A_{D}\right\}$ and the representation

$$
\xi_{B}(\lambda)=\sum_{j \in J} \lim _{\varepsilon \downarrow 0} \pi^{-1}\left(\operatorname{Im}\left(\log \left(\mathfrak{N}_{V}(\lambda+i \varepsilon)\right)\right) \varphi_{j}, \varphi_{j}\right)_{L^{2}(\mathcal{S})}
$$

for a spectral shift function $\xi_{B}$ of the pair $\left\{B, B_{D}\right\}$.

Step 1 In this step we consider the operators $B$ and $B_{D}$ as self-adjoint extensions of the closed symmetric $S=B \cap B_{D}$, which is given by

$$
S=-\Delta+V, \quad \operatorname{dom}(S)=\left\{f \in H^{2}\left(\mathbb{R}^{n}\right) \mid \gamma_{D}^{+} f_{+}=0=\gamma_{D}^{-} f_{-}\right\}
$$

Furthermore, consider the operator

$$
T=-\Delta+V, \quad \operatorname{dom}(T)=\left\{f=\left(\begin{array}{c}
f_{+} \\
f_{-}
\end{array}\right) \in H^{2}\left(\mathcal{B}_{+}\right) \times H^{2}\left(\mathcal{B}_{-}\right) \mid \gamma_{D}^{+} f_{+}=\gamma_{D}^{-} f_{-}\right\}
$$

and set $\gamma_{D} f:=\gamma_{D}^{+} f_{+}=\gamma_{D}^{-} f_{-}$for $f \in \operatorname{dom}(T)$. It is easy to see with the help of Theorem 2.2, (6.4)-(6.5) and (5.3) that $\left\{L^{2}(\mathcal{S}), \Gamma_{0}, \Gamma_{1}\right\}$, where

$$
\Gamma_{0} f=\imath^{-1}\left(\gamma_{N}^{+} f_{+}+\gamma_{N}^{-} f_{-}\right) \quad \text { and } \quad \Gamma_{1} f=\imath \gamma_{D} f, \quad f \in \operatorname{dom}(T),
$$

is a quasi boundary triple for $T \subset S^{*}$ and $B=T \uparrow \operatorname{ker}\left(\Gamma_{0}\right)$ and $B_{D}=T \uparrow \operatorname{ker}\left(\Gamma_{1}\right)$ hold (cf. the proof of [8, Theorem 5.1]). The corresponding Weyl function is

$$
M(z) \varphi=\imath\left(\mathcal{D}_{+}^{V}(z)+\mathcal{D}_{-}(z)\right)^{-1} \tilde{\imath} \varphi=\mathfrak{N}_{V}(z) \varphi
$$

for all $z \in \rho(B) \cap \rho\left(B_{D}\right)$ and $\varphi \in \operatorname{ran}\left(\Gamma_{0}\right)$. Furthermore, the proof of [8, Theorem 5.1] shows that $M(z)$ and $M(z)^{-1}$ are bounded for all $z \in \rho(B) \cap \rho\left(B_{D}\right)$ and one has $\overline{M(z)}=\mathfrak{N}_{V}(z)$.

One observes that $B$ corresponds to the densely defined, closed quadratic form

$$
\mathfrak{b}[f, g]=(\nabla f, \nabla g)_{\left(L^{2}\left(\mathbb{R}^{n}\right)\right)^{n}}+(V f, g)_{L^{2}\left(\mathbb{R}^{n}\right)}, \quad \operatorname{dom}(\mathfrak{b})=H^{1}\left(\mathbb{R}^{n}\right),
$$

and that $B_{D}$ corresponds to the densely defined closed quadratic form

$$
\mathfrak{b}_{D}[f, g]=(\nabla f, \nabla g)_{\left(L^{2}\left(\mathbb{R}^{n}\right)\right)^{n}}+(V f, g)_{L^{2}\left(\mathbb{R}^{n}\right)}, \quad \operatorname{dom}\left(\mathfrak{b}_{D}\right)=H_{0}^{1}\left(\mathcal{B}_{+}\right) \times H_{0}^{1}\left(\mathcal{B}_{-}\right)
$$

Since $H^{1}\left(\mathbb{R}^{n}\right) \subset\left(H_{0}^{1}\left(\mathcal{B}_{+}\right) \times H_{0}^{1}\left(\mathcal{B}_{-}\right)\right)$this implies $\mathfrak{b} \leq \mathfrak{b}_{D}$ and yields the sign condition $\left(B-\zeta I_{L^{2}\left(\mathbb{R}^{n}\right)}\right)^{-1} \geq\left(B_{D}-\zeta I_{L^{2}\left(\mathbb{R}^{n}\right)}\right)^{-1}$ in the assumptions of Theorem 4.1 
for all $\zeta<\min (\sigma(B)) \leq \min \left(\sigma\left(B_{D}\right)\right)$; see the beginning of Step 2 in the proof of Theorem 5.3.

Next, we verify the $\mathfrak{S}_{p}$-conditions

$$
\begin{aligned}
& \overline{\gamma(z)}^{(p)}\left(M(z)^{-1} \gamma(\bar{z})^{*}\right)^{(q)} \in \mathfrak{S}_{1}\left(L^{2}\left(\mathbb{R}^{n}\right)\right), \quad p+q=2 k, \\
& \left(M(z)^{-1} \gamma(\bar{z})^{*}\right)^{(q)} \overline{\gamma(z)}{ }^{(p)} \in \mathfrak{S}_{1}\left(L^{2}(\mathcal{S})\right), \quad p+q=2 k,
\end{aligned}
$$

and

$$
\frac{d^{j}}{d z^{j}} \overline{M(z)} \in \mathfrak{S}_{(2 k+1) / j}\left(L^{2}(\mathcal{S})\right), \quad j=1, \ldots, 2 k+1,
$$

for all $z \in \rho(B) \cap \rho\left(B_{D}\right)$ in the assumptions of Theorem 4.1. For this we use the smoothing property

$$
\left(B-z I_{L^{2}\left(\mathbb{R}^{n}\right)}\right)^{-1} f \in H_{\mathcal{O}}^{\ell+2}\left(\mathbb{R}^{n}\right) \quad \text { for all } f \in H_{\mathcal{O}}^{\ell}\left(\mathbb{R}^{n}\right) \text { and } \quad \ell \in \mathbb{N}_{0},
$$

where $\mathcal{O}$ is an open neighborhood of the sphere $\mathcal{S}$ in $\mathbb{R}^{n}$ such that $\operatorname{supp}(V) \cap \mathcal{O}=\varnothing$ and $H_{\mathcal{O}}^{\ell}\left(\mathbb{R}^{n}\right)=\left\{f \in L^{2}\left(\mathbb{R}^{n}\right) \mid f\left\lceil\mathcal{O} \in H^{\ell}(\mathcal{O})\right\}\right.$ (cf. [5, Lemma 4.1(i)]).

It follows from (2.2) and the definition of $\Gamma_{1}$ that

$$
\gamma(\bar{z})^{*} f=\Gamma_{1}\left(B-z I_{L^{2}\left(\mathbb{R}^{n}\right)}\right)^{-1} f=\imath \gamma_{D}\left(B-z I_{L^{2}\left(\mathbb{R}^{n}\right)}\right)^{-1} f
$$

and hence (2.6) yields

$$
\left(\gamma(\bar{z})^{*}\right)^{(q)}=q ! \gamma(\bar{z})^{*}\left(B-z I_{L^{2}\left(\mathbb{R}^{n}\right)}\right)^{-q}=q ! \iota \gamma_{D}\left(B-z I_{L^{2}\left(\mathbb{R}^{n}\right)}\right)^{-(q+1)} .
$$

Since

$$
\operatorname{ran}\left(\gamma_{D}\left(B-z I_{L^{2}\left(\mathbb{R}^{n}\right)}\right)^{-(q+1)}\right) \subset H^{2 q+(3 / 2)}(\mathcal{S})
$$

by (6.17) and (5.5), and the operator $\gamma_{D}\left(B-z I_{L^{2}\left(\mathbb{R}^{n}\right)}\right)^{-(q+1)}$ is bounded from $L^{2}\left(\mathbb{R}^{n}\right)$ into $H^{1 / 2}(\mathcal{S})$ it follows from Lemma 5.2 with $s=2 q+(3 / 2)$ and $t=1 / 2$ that

$$
\gamma_{D}\left(B-z I_{L^{2}\left(\mathbb{R}^{n}\right)}\right)^{-(q+1)} \in \mathfrak{S}_{r}\left(L^{2}\left(\mathbb{R}^{n}\right), H^{1 / 2}(\mathcal{S})\right) . \quad r>(n-1) /(2 q+1),
$$

As $\imath: H^{1 / 2}(\mathcal{S}) \rightarrow L^{2}(\mathcal{S})$ is an isomorphism one concludes from (6.19) that

$$
\left(\gamma(\bar{z})^{*}\right)^{(q)} \in \mathfrak{S}_{r}\left(L^{2}\left(\mathbb{R}^{n}\right), L^{2}(\mathcal{S})\right), \quad r>(n-1) /(2 q+1),
$$

for all $z \in \rho(B)$ and $q \in \mathbb{N}_{0}$. From this it is also clear that

$$
\overline{\gamma(z)}^{(p)} \in \mathfrak{S}_{r}\left(L^{2}(\mathcal{S}), L^{2}\left(\mathbb{R}^{n}\right)\right), \quad r>(n-1) /(2 p+1),
$$

for all $z \in \rho(B)$ and $p \in \mathbb{N}_{0}$. Furthermore,

$$
\frac{d^{j}}{d z^{j}} \overline{M(z)}=j ! \gamma(\bar{z})^{*}\left(B-z I_{L^{2}\left(\mathbb{R}^{n}\right)}\right)^{-(j-1)} \overline{\gamma(z)}, \quad j \in \mathbb{N},
$$


by (2.12), and using (6.18) one obtains with the arguments above that

$$
\gamma(\bar{z})^{*}\left(B-z I_{L^{2}\left(\mathbb{R}^{n}\right)}\right)^{-(j-1)}=\imath \gamma_{D}\left(B-z I_{L^{2}\left(\mathbb{R}^{n}\right)}\right)^{-j} \in \mathfrak{S}_{r}\left(L^{2}\left(\mathbb{R}^{n}\right), L^{2}(\mathcal{S})\right)
$$

for $r>(n-1) /(2 j-1), z \in \rho(B)$, and $j \in \mathbb{N}$. Together with (6.21) for $p=0$ one finds that (6.22) satisfies

$$
\frac{d^{j}}{d z^{j}} \overline{M(z)} \in \mathfrak{S}_{r}\left(L^{2}(\mathcal{S})\right), \quad r>(n-1) /(2 j),
$$

for all $z \in \rho(B)$ and $j \in \mathbb{N}$. The same arguments as in Step 3 of the proof Theorem 5.3 show that

$$
\frac{d^{j}}{d z^{j}} \overline{M(z)}-1 \in \mathfrak{S}_{r}\left(L^{2}(\mathcal{S})\right), \quad r>(n-1) /(2 j),
$$

for all $z \in \rho(B) \cap \rho\left(B_{D}\right)$ and $j \in \mathbb{N}$. It follows from (6.20) and (6.24) that each summand in the right-hand side in

$$
\left(M(z)^{-1} \gamma(\bar{z})^{*}\right)^{(q)}=\sum_{\substack{p+m=q \\
p, m \geq 0}}\left(\begin{array}{l}
q \\
p
\end{array}\right)\left(\overline{M(z)}^{-1}\right)^{(p)}\left(\gamma(\bar{z})^{*}\right)^{(m)}
$$

belongs to $\mathfrak{S}_{r}\left(L^{2}(\mathcal{S}), L^{2}\left(\mathbb{R}^{n}\right)\right)$ for $r>(n-1) /(2 q+1)$, and hence one infers together with (6.21) that

$$
\overline{\gamma(z)}^{(p)}\left(M(z)^{-1} \gamma(\bar{z})^{*}\right)^{(q)} \in \mathfrak{S}_{r}\left(L^{2}\left(\mathbb{R}^{n}\right)\right)
$$

for $r>(n-1) /[2(p+q)+2]=(n-1) /(4 k+2)$, and since $k>(n-2) / 4$ by assumption, one has $1>(n-1) /(4 k+2)$, implying the trace class condition (6.14). The same argument shows that (6.15) is satisfied. Finally, (6.16) follows from (6.23) and the fact that $k>(n-2) / 4$ implies

$$
\frac{2 k+1}{j}>\frac{n}{2 j}>\frac{n-1}{2 j}, \quad j=1, \ldots, 2 k+1 .
$$

Hence, the assumptions in Theorem 4.1 are satisfied with $S$ in (6.11), the quasi boundary triple in (6.12), and the corresponding Weyl function in (6.13). Thus, The- 
orem 4.1 yields assertion $(i)$ with $A$ replaced by $B_{D}$ and for any orthonormal basis $\left(\varphi_{j}\right)_{j \in J}$ in $L^{2}(\mathcal{S})$ the function

$$
\xi_{B}(\lambda)=\sum_{j \in J} \lim _{\varepsilon \downarrow 0} \pi^{-1}\left(\operatorname{Im}\left(\log \left(\mathfrak{N}_{V}(\lambda+i \varepsilon)\right)\right) \varphi_{j}, \varphi_{j}\right)_{L^{2}(\mathcal{S})} \quad \text { for a.e. } \lambda \in \mathbb{R}
$$

in (6.10) is a spectral shift function for the pair $\left\{B, B_{D}\right\}$ and the trace formula

$$
\operatorname{tr}_{L^{2}\left(\mathbb{R}^{n}\right)}\left(\left(B_{D}-z I_{L^{2}\left(\mathbb{R}^{n}\right)}\right)^{-(2 k+1)}-\left(B-z I_{L^{2}\left(\mathbb{R}^{n}\right)}\right)^{-(2 k+1)}\right)=-(2 k+1) \int_{\mathbb{R}} \frac{\xi_{B}(\lambda) d \lambda}{(\lambda-z)^{2 k+2}}
$$

is valid for all $z \in \rho(B) \cap \rho\left(B_{D}\right)$.

Step 2 Now we complete the proof of Theorem 6.1. First, we note that the same arguments as in Step 1 with $V=0$ show that assertion $(i)$ in Theorem 6.1 holds with $B$ replaced by $A_{D}$ and $\xi_{A}$ in (6.9) is a spectral shift function for the pair $\left\{A, A_{D}\right\}$ such that

$$
\operatorname{tr}_{L^{2}\left(\mathbb{R}^{n}\right)}\left(\left(A_{D}-z I_{L^{2}\left(\mathbb{R}^{n}\right)}\right)^{-(2 k+1)}-\left(A-z I_{L^{2}\left(\mathbb{R}^{n}\right)}\right)^{-(2 k+1)}\right)=-(2 k+1) \int_{\mathbb{R}} \frac{\xi_{A}(\lambda) d \lambda}{(\lambda-z)^{2 k+2}}
$$

holds for all $z \in \mathbb{C} \backslash[0, \infty)$. The assumption $k>(n-2) / 4$ implies $2 k+1>n / 2$ and hence

$$
\begin{aligned}
& \left(A_{+}-z I_{L^{2}\left(\mathcal{B}_{+}\right)}\right)^{-(2 k+1)} \in \mathfrak{S}_{1}\left(L^{2}\left(\mathcal{B}_{+}\right)\right), \quad z \in \rho\left(A_{+}\right), \\
& \left(B_{+}-\zeta I_{L^{2}\left(\mathcal{B}_{+}\right)}\right)^{-(2 k+1)} \in \mathfrak{S}_{1}\left(L^{2}\left(\mathcal{B}_{+}\right)\right), \quad \zeta \in \rho\left(B_{+}\right),
\end{aligned}
$$

by standard Weyl asymptotics. Furthermore, since the spectra of $A_{+}$and $B_{+}$are discrete and bounded from below, it is well-known that

$$
\xi_{+}(\lambda)=N\left(\lambda, B_{+}\right)-N\left(\lambda, A_{+}\right), \quad \lambda \in \mathbb{R},
$$

is a spectral shift function for the pair $\left\{A_{+}, B_{+}\right\}$(see, e.g., [16, (3.28)]). From (6.8) it is clear that $\xi_{+}$is also a spectral shift function for the pair $\left\{A_{D}, B_{D}\right\}$. Since

$$
\left[\left(B_{D}-z I_{L^{2}\left(\mathbb{R}^{n}\right)}\right)^{-(2 k+1)}-\left(A_{D}-z I_{L^{2}\left(\mathbb{R}^{n}\right)}\right)^{-(2 k+1)}\right] \in \mathfrak{S}_{1}\left(L^{2}\left(\mathbb{R}^{n}\right)\right)
$$

by (6.27) and (6.8) one concludes that

$$
\operatorname{tr}_{L^{2}\left(\mathbb{R}^{n}\right)}\left(\left(B_{D}-z I_{L^{2}\left(\mathbb{R}^{n}\right)}\right)^{-(2 k+1)}-\left(A_{D}-z I_{L^{2}\left(\mathbb{R}^{n}\right)}\right)^{-(2 k+1)}\right)=-(2 k+1) \int_{\mathbb{R}} \frac{\xi_{+}(\lambda) d \lambda}{(\lambda-z)^{2 k+2}}
$$

for $z \in \rho\left(A_{D}\right) \cap \rho\left(B_{D}\right)$. Hence, $\xi(\lambda)=\xi_{A}(\lambda)-\xi_{B}(\lambda)+\xi_{+}(t)$ for a.e. $\lambda \in \mathbb{R}$ is a spectral shift function for the pair $\{A, B\}$, and taking into account the specific form of $\xi_{A}, \xi_{B}$, and $\xi_{+}$, in (6.9), (6.10), and (6.28) and the trace formulas (6.25), (6.26), and (6.29), the assertions in Theorem 6.1 follow. 
Remark 6.2 We note that the spectral shift function $\xi$ in Theorem 6.1 is continuous for $\lambda>0$ since $V \in L^{\infty}\left(\mathbb{R}^{n}\right)$ is compactly supported (see, e.g., [76, Theorem 9.1.20]). On the other hand the spectral shift function $\xi_{+}$of $\left\{A_{+}, B_{+}\right\}$is a step function and hence the difference of the spectral shift functions $\xi_{A}$ and $\xi_{B}$ of the pairs $\left\{A, A_{D}\right\}$ and $\left\{B, B_{D}\right\}$ cancel the discontinuities of $\xi_{+}$for $\lambda>0$.

Acknowledgements Open access funding provided by Graz University of Technology. The authors wish to thank the referees for helpful comments and remarks that led to an improvement of the manuscript. Jussi Behrndt is most grateful for the stimulating research stay and the hospitality at the Graduate School of Mathematical Sciences of the University of Tokyo from April to July 2016, where parts of this paper were written. The authors also wish to thank Hagen Neidhardt for fruitful discussions and helpful remarks. This work is supported by International Relations and Mobility Programs of the TU Graz and the Austrian Science Fund (FWF), Project P 25162-N26.

Open Access This article is distributed under the terms of the Creative Commons Attribution 4.0 International License (http://creativecommons.org/licenses/by/4.0/), which permits unrestricted use, distribution, and reproduction in any medium, provided you give appropriate credit to the original author(s) and the source, provide a link to the Creative Commons license, and indicate if changes were made.

\section{References}

1. Azamov, N.A., Carey, A.L., Dodds, P.G., Sukochev, F.A.: Operator integrals, spectral shift, and spectral flow. Can. J. Math. 61, 241-263 (2009)

2. Behrndt, J., Langer, M.: Boundary value problems for elliptic partial differential operators on bounded domains. J. Funct. Anal. 243, 536-565 (2007)

3. Behrndt, J., Langer, M.: Elliptic operators, Dirichlet-to-Neumann maps and quasi boundary triples. In: Operator Methods for Boundary Value Problems, London Math. Soc. Lecture Note Series, vol. 404, pp. 121-160 (2012)

4. Behrndt, J., Langer, M., Lobanov, I., Lotoreichik, V., Popov, IYu.: A remark on Schatten-von Neumann properties of resolvent differences of generalized Robin Laplacians on bounded domains. J. Math. Anal. Appl. 371, 750-758 (2010)

5. Behrndt, J., Langer, M., Lotoreichik, V.: Spectral estimates for resolvent differences of self-adjoint elliptic operators. Integr. Equations Oper. Theory 77, 1-37 (2013)

6. Behrndt, J., Langer, M., Lotoreichik, V.: Trace formulae and singular values of resolvent power differences of self-adjoint elliptic operators. J. Lond. Math. Soc. 88, 319-337 (2013)

7. Behrndt, J., Malamud, M.M., Neidhardt, H.: Scattering matrices and Weyl functions. Proc. Lond. Math. Soc. 97, 568-598 (2008)

8. Behrndt, J., Malamud, M.M., Neidhardt, H.: Scattering matrices and Dirichlet-to-Neumann maps. J. Funct. Anal. 273, 1970-2025 (2017)

9. Birman, M.Sh.: Perturbations of the continuous spectrum of a singular elliptic operator by varying the boundary and the boundary conditions. Vestnik Leningrad. Univ. 17, 22-55 (1962)

10. Birman, M.Sh., Entina, S.B.: Stationary approach in abstract scattering theory, Izv. Akad. Nauk SSSR Ser. Mat. 31 (1967), 401-430, translation in Math. USSR Izv. 1, no. 1, (1967), 391-420

11. Birman, M.Sh., Krein, M.G.: On the theory of wave operators and scattering operators. Dokl. Akad. Nauk SSSR 144, 475-478 (1962)

12. Birman, M.Sh., Krein, M.G.: Some topics of the theory of the wave and scattering operators, 1963 Outlines Joint Sympos. Partial Differential Equations (Novosibirsk, 1963). Acad, Sci. USSR Siberian Branch. Moscow, pp. 39-45 (1963)

13. Birman, M.Sh., Pushnitski, A.B.: Spectral shift function, amazing and multifaceted. Integr. Equations Oper. Theory 30, 191-199 (1998)

14. Birman, M.Sh., Solomyak, M.Z.: Remarks on the spectral shift function. J. Sov. Math. 3, 408-419 (1975)

15. Birman, M.Sh., Solomjak, M.Z.: Spectral Theory of Self-Adjoint Operators in Hilbert Spaces. D. Reidel Publishing Co., Dordrecht (1987) 
16. Birman, M.Sh., Yafaev, D.R.: The spectral shift function. The papers of M. G. Krein and their further development. Algebra i Analiz 4(5), 1-44 (1992) [translation in St. Petersburg Math. J. 4 (1993), no. 5, 833-870]

17. Birman, M.Sh., Yafaev, D.R.: Spectral properties of the scattering matrix. Algebra i Analiz 4(6) 1-27 (1992) [translation in St. Petersburg Math. J. 4 (1993), no. 6, 1055-1079]

18. Brüning, J., Geyler, V., Pankrashkin, K.: Spectra of self-adjoint extensions and applications to solvable Schrödinger operators. Rev. Math. Phys. 20, 1-70 (2008)

19. Carey, R.W.: A unitary invariant for pairs of self-adjoint operators. J. Reine Angew. Math. 283/284, 294-312 (1976)

20. Deift, P., Simon, B.: On the decoupling of finite singularities from the question of asymptotic completeness in two body quantum systems. J. Funct. Anal. 23, 218-238 (1976)

21. Derkach, V.A., Hassi, S., Malamud, M.M., de Snoo, H.S.V.: Boundary relations and their Weyl families. Trans. Am. Math. Soc. 358, 5351-5400 (2006)

22. Derkach, V.A., Malamud, M.M.: Generalized resolvents and the boundary value problems for Hermitian operators with gaps. J. Funct. Anal. 95, 1-95 (1991)

23. Derkach, V.A., Malamud, M.M.: The extension theory of Hermitian operators and the moment problem. J. Math. Sci. (NY) 73, 141-242 (1995)

24. Gesztesy, F., Holden, H., Simon, B.: Absolute summability of the trace relation for certain Schrödinger operators. Commun. Math. Phys. 168, 137-161 (1995)

25. Gesztesy, F., Latushkin, Yu., Makarov, K.A., Sukochev, F.A., Tomilov, Yu.: The index formula and the spectral shift function for relatively trace class perturbations. Adv. Math. 227, 319-420 (2011)

26. Gesztesy, F., Makarov, K.A., Naboko, S.N.: The spectral shift operator. Oper. Theory Adv. Appl. 108, 59-90 (1999)

27. Gesztesy, F., Makarov, K.A.: The $\Xi$ operator and its relation to Krein's spectral shift function. J. Anal. Math. 81, 139-183 (2000)

28. Gesztesy, F., Mitrea, M.: Generalized Robin boundary conditions, Robin-to-Dirichlet maps, Kreŭn-type resolvent formulas for Schrödinger operators on bounded Lipschitz domains. Proc. Symp. Pure Math. Am. Math. Soc. 79, 105-173 (2008)

29. Gesztesy, F., Mitrea, M.: A description of all self-adjoint extensions of the Laplacian and Krein-type resolvent formulas on non-smooth domains. J. Anal. Math. 113, 53-172 (2011)

30. Gesztesy, F., Nichols, R.: An abstract approach to weak convergence of spectral shift functions and applications to multi-dimensional Schrödinger operators. J. Spectral Theory 2, 225-266 (2012)

31. Gesztesy, F., Simon, B.: The xi function. Acta Math. 176, 49-71 (1996)

32. Gorbachuk, V.I., Gorbachuk, M.L.: Boundary Value Problems for Operator Differential Equations. Kluwer Academic Publishers, Dordrecht (1991)

33. Grubb, G.: Singular Green operators and their spectral asymptotics. Duke Math. J. 51, 477-528 (1984)

34. Grubb, G.: Spectral asymptotics for Robin problems with a discontinuous coefficient. J. Spectral Theory 1, 155-177 (2011)

35. Grubb, G.: The mixed boundary value problem, Kreı̆n resolvent formulas and spectral asymptotic estimates. J. Math. Anal. Appl. 382, 339-363 (2011)

36. Grubb, G.: Perturbation of essential spectra of exterior elliptic problems. Appl. Anal. 90, 103-123 (2011)

37. Grubb, G.: Krein-like extensions and the lower boundedness problem for elliptic operators. J. Differ. Equations 252, 852-885 (2012)

38. Hassi, S., de Snoo, H.S.V., Woracek, H.: Some interpolation problems of Nevanlinna-Pick type. KreinLanger method. Oper. Theory Adv. Appl. 106, 201-216 (1998)

39. Hislop, P.D., Müller, P.: The spectral shift function for compactly supported perturbations of Schrödinger operators on large bounded domains. Proc. Am. Math. Soc. 138, 2141-2150 (2010)

40. Hundertmark, D., Killip, R., Nakamura, S., Stollmann, P., Veselić, I.: Bounds on the spectral shift function and the density of states. Commun. Math. Phys. 262, 489-503 (2006)

41. Kato, T.: Perturbation Theory for Linear Operators, Grundlehren der mathematischen Wissenschaften, vol. 132. Springer, Berlin, New York (1976)

42. Kohmoto, M., Koma, T., Nakamura, S.: The spectral shift function and the Friedel sum rule. Ann. Henri Poincaré 14, 1413-1424 (2013)

43. Koplienko, L.S.: The spectrum of the scattering matrix. Izv. Vyss. Ucebn. Zaved. Matematika 108(5), 54-61 (1971)

44. Krein, M.G.: On the trace formula in perturbation theory. Mat. Sbornik 33, 597-626 (1953) 
45. Krein, M.G.: On perturbation determinants and a trace formula for unitary and self-adjoint operators. Dokl. Akad. Nauk SSSR 144, 268-271 (1962)

46. Krein, M.G.: Perturbation determinants and a trace formula for some classes of pairs of operators. J. Oper. Theory 17, 129-187 (1987)

47. Krein, M.G., Yavryan, V.A.: Spectral shift functions that arise in perturbations of a positive operator. J. Oper. Theory 6, 155-191 (1981)

48. Krüger, H., Teschl, G.: Relative oscillation theory, weighted zeros of the Wronskian, and the spectral shift function. Commun. Math. Phys. 287, 613-640 (2009)

49. Langer, H., de Snoo, H.S.V., Yavrian, V.A.: A relation for the spectral shift function of two selfadjoint extensions. Oper. Theory Adv. Appl. 127, 437-445 (2001)

50. Langer, H., Textorius, B.: On generalized resolvents and $Q$-functions of symmetric linear relations (subspaces) in Hilbert space. Pac. J. Math. 72, 135-165 (1977)

51. Lifshitz, I.M.: On a problem of the theory of perturbations connected with quantum statistics. Uspehi Matem. Nauk 7, 171-180 (1952)

52. Lifshitz, I.M.: Some problems of the dynamic theory of nonideal crystal lattices. Nuovo Cimento Suppl. (Ser. X) 3, 716-734 (1956)

53. Lions, J., Magenes, E.: Non-Homogeneous Boundary Value Problems and Applications I. Springer, Berlin, Heidelberg, New York (1972)

54. Malamud, M.M.: Spectral theory of elliptic operators in exterior domains. Russ. J. Math. Phys. 17, 96-125 (2010)

55. Malamud, M.M., Neidhardt, H.: Perturbation determinants for singular perturbations. Russ. J. Math. Phys. 21, 55-98 (2014)

56. Malamud, M.M., Neidhardt, H.: Trace formulas for additive and non-additive perturbations. Adv. Math. 274, 736-832 (2015)

57. Mantile, A., Posilicano, A., Sini, M.: Self-adjoint elliptic operators with boundary conditions on not closed hypersurfaces. J. Differ. Equations 261, 1-55 (2016)

58. Mantile, A., Posilicano, A., Sini, M.: Limiting absorption principle, generalized eigenfunction expansions and scattering matrix for Laplace operators with boundary conditions on hypersurfaces. J. Spectral Theory (2017) (to appear)

59. McLean, W.: Strongly Elliptic Systems and Boundary Integral Equations. Cambridge University Press, Cambridge (2000)

60. Naboko, S.N.: Uniqueness theorems for operator-valued functions with positive imaginary part, and the singular spectrum in the Friedrichs model. Ark. Mat. 25, 115-140 (1987)

61. Naboko, S.N.: Boundary values of analytic operator functions with a positive imaginary part. J. Sov. Math. 44, 786-795 (1989)

62. Naboko, S.N.: Nontangential boundary values of operator-valued $R$-functions in a half-plane. Leningrad Math. J. 1, 1255-1278 (1990)

63. Post, O.: Boundary pairs associated with quadratic forms. Math. Nachr. 289, 1052-1099 (2016)

64. Potapov, D., Skripka, A., Sukochev, F.A.: Spectral shift function of higher order. Invent. Math. 193, 501-538 (2013)

65. Potapov, D., Skripka, A., Sukochev, F.A.: Higher-order spectral shift for contractions. Proc. Lond. Math. Soc. 108, 327-349 (2014)

66. Potapov, D., Sukochev, F.A.: Koplienko spectral shift function on the unit circle. Commun. Math. Phys. 309, 693-702 (2012)

67. Pushnitski, A.B.: A representation for the spectral shift function in the case of perturbations of fixed sign. Algebra i Analiz 9, 197-213 (1997) [translation in St. Petersburg Math. J. 9(1998), 1181-1194]

68. Pushnitski, A.B.: The spectral flow, the Fredholm index, and the spectral shift function. In: Spectral theory of differential operators, Amer. Math. Soc. Transl. Ser. 2, vol. 225. American Mathematical Society, Providence, pp. 141-155 (2008)

69. Schmüdgen, K.: Unbounded Self-Adjoint Operators on Hilbert Space. Springer, Dordrecht (2012)

70. Simon, B.: Spectral averaging and the Krein spectral shift. Proc. Am. Math. Soc. 126, 1409-1413 (1998)

71. Sjöstrand, J., Zworski, M.: Complex scaling and the distribution of scattering poles. J. Am. Math. Soc. 4, 729-769 (1991)

72. Weidmann, J.: Linear Operators in Hilbert Spaces, Graduate Texts in Mathematics, vol. 68. Springer, New York (1980) 
73. Yafaev, D.R.: Mathematical Scattering Theory. General Theory, Translations of Mathematical Monographs, vol. 105. American Mathematical Society, Providence (1992)

74. Yafaev, D.R.: A trace formula for the Dirac operator. Bull. Lond. Math. Soc. 37, 908-918 (2005)

75. Yafaev, D.R.: The Schrödinger operator: perturbation determinants, the spectral shift function, trace identities, and more. Funktsional. Anal. i Prilozhen. 41, 60-83, 96 (2007) [translation in Funct. Anal. Appl. 41(2007), 217-236]

76. Yafaev, D.R.: Mathematical Scattering Theory. Analytic Theory, Mathematical Surveys and Monographs, vol. 158. American Mathematical Society, Providence (2010) 